\title{
The MBHBM ${ }^{\star}$ Project - II. Molecular gas kinematics in the lenticular galaxy NGC 3593 reveal a supermassive black hole
}

\author{
Dieu D. Nguyen ${ }^{\oplus}, 1,2 \star$ Martin Bureau, ${ }^{3,4}$ Sabine Thater, ${ }^{5}$ Kristina Nyland, ${ }^{6}$ Mark den Brok, ${ }^{7}$ \\ Michele Cappellari ${ }^{\circledR},{ }^{3}$ Timothy A. Davis ${ }^{\circledR},{ }^{8}$ Jenny E. Greene, ${ }^{9}$ Nadine Neumayer, ${ }^{10}$ \\ Masatoshi Imanishi ${ }^{\circledR}$, 11,12 Takuma Izumi ${ }^{\circledR}, 11,12$ Taiki Kawamuro, ${ }^{13}$ Shunsuke Baba, ${ }^{11}$ \\ Phuong M. Nguyen, ${ }^{14}$ Satoru Iguchi ${ }^{\circledR},{ }^{11,12}$ Takafumi Tsukui, ${ }^{11,12}$ Lam N. T. ${ }^{15}$ and Than Ho ${ }^{16}$ \\ Affiliations are listed at the end of the paper
}

Accepted 2021 October 15. Received 2021 October 6; in original form 2021 March 11

\begin{abstract}
As part of the Measuring Black Holes in below Milky Way-mass $\left(\mathrm{M}^{\star}\right)$ galaxies $\left(\mathrm{MBHBM}^{\star}\right)$ Project, we present a dynamical measurement of the supermassive black hole (SMBH) mass in the nearby lenticular galaxy NGC 3593, using cold molecular gas ${ }^{12} \mathrm{CO}(2-1)$ emission observed at an angular resolution of $\approx 0 ! 3(\approx 10 \mathrm{pc})$ with the Atacama Large Millimeter/submillimeter Array (ALMA). Our ALMA observations reveal a circumnuclear molecular gas disc (CND) elongated along the galaxy major axis and rotating around the SMBH. This CND has a relatively low-velocity dispersion $\left(\lessgtr 10 \mathrm{~km} \mathrm{~s}^{-1}\right)$ and is morphologically complex, with clumps having higher integrated intensities and velocity dispersions $\left(\$ 25 \mathrm{~km} \mathrm{~s}^{-1}\right)$. These clumps are distributed along the ridges of a two-arm/bi-symmetric spiral pattern surrounded by a larger ring-like structure (radius $r \approx 10 \operatorname{arcsec}$ or $\approx 350 \mathrm{pc}$ ). This pattern likely plays an important role to bridge the molecular gas reservoirs in the CND and beyond $(10 \lesssim r \lessgtr$ 35 arcsec or $350 \mathrm{pc} \lesssim r \lesssim 1.2 \mathrm{kpc}$ ). Using dynamical modelling, the molecular gas kinematics allow us to infer an SMBH mass $M_{\mathrm{BH}}=2.40_{-1.05}^{+1.87} \times 10^{6} \mathrm{M}_{\odot}$ (only statistical uncertainties at the $3 \sigma$ level). We also detect a massive core of cold molecular gas $(\mathrm{CMC})$ of mass $M_{\mathrm{CMC}}=(5.4 \pm 1.2) \times 10^{6} \mathrm{M}_{\odot}$ and effective (half-mass) radius $r_{\mathrm{CMC}, \mathrm{e}}=11.2 \pm 2.8 \mathrm{pc}$, co-spatial with a nuclear star cluster $(\mathrm{NSC})$ of mass $M_{\mathrm{NSC}}=(1.67 \pm 0.48) \times 10^{7} \mathrm{M}_{\odot}$ and effective radius $r_{\mathrm{NSC}, \mathrm{e}}=5.0 \pm 1.0 \mathrm{pc}\left(\right.$ or $\left.0{ }^{\prime} \cdot 15 \pm 00^{\prime} 03\right)$. The mass profiles of the CMC and NSC are well described by Sérsic functions with indices 1-1.4. Our $M_{\mathrm{BH}}$ and $M_{\mathrm{NSC}}$ estimates for NGC 3593 agree well with the recently compiled $M_{\mathrm{BH}}-M_{\mathrm{NSC}}$ scaling relation. Although the $M_{\mathrm{NSC}}$ uncertainty is twice the inferred $M_{\mathrm{BH}}$, the rapid central rise of the rotation velocities of the CND (as the radius decreases) clearly suggests an SMBH. Indeed, our dynamical models show that even if $M_{\mathrm{NSC}}$ is at the upper end of its allowed range, the evidence for a $\mathrm{BH}$ does not vanish, but remains with a lower limit of $M_{\mathrm{BH}}>3 \times 10^{5} \mathrm{M}_{\odot}$.
\end{abstract}

Key words: Galaxy: disc-galaxies: ISM - galaxies: kinematics and dynamics - galaxies: nuclei - ISM: molecules - (galaxies:) quasars: supermassive black holes.

\section{INTRODUCTION}

The co-evolution of supermassive black holes (SMBHs) and their host galaxies is one of the most important puzzles in galaxy formation and evolution (e.g. Schawinski et al. 2007). Galaxies with spheroids (or more generally bulges) ubiquitously harbour SMBHs at their centres, and the SMBH masses $\left(M_{\mathrm{BH}}\right)$ correlate surprisingly well with macroscopic properties of the bulges, e.g. central stellar velocity dispersion $\left(\sigma_{\star}\right.$; Ferrarese \& Merritt 2000; Gebhardt et al. 2000) and stellar mass ( $M_{\text {bulge }}$; Kormendy \& Richstone 1995; Magorrian et al. 1998; Marconi \& Hunt 2003; Häring \& Rix 2004), despite bulges extending far beyond the sphere of influence (SOI) of even the largest black hole $\left(\mathrm{BH}^{1}\right)$.

The tightest scaling relation among these relationships is the $M_{\mathrm{BH}}-$ $\sigma_{\star}$ correlation (e.g. Ferrarese \& Merritt 2000; Gebhardt et al. 2000),

\footnotetext{
^E-mail: nddieuphys@gmail.com,nddieu@hcmiu.edu.vn

${ }^{1}$ In this work, we use the abbreviations SMBH and BH interchangeably.
}

but there is growing evidence of divergence between galaxies of different morphological types or bulge masses, especially towards the low-mass regimes of both BHs and their hosts (see e.g. fig. 1 of Krajnović et al. 2018 and fig. 17 of Nguyen et al. 2019). To fully understand the extent of the co-evolution among all these galaxy properties, it is essential to gather a larger, more diverse sample of low-mass galaxies and perform more reliable measurements of their SMBH masses (e.g. McConnell et al. 2013; van den Bosch et al. 2016; Graham et al. 2018; Nguyen et al. 2018, 2019).

Recently, the number of known $\lesssim 10^{6} \mathrm{M}_{\odot}$ BHs has increased dramatically, with masses inferred from a variety of methods including (1) the velocity widths of broad optical emission lines (Barth et al. 2004; Greene \& Ho 2007; Thornton et al. 2008; Dong et al. 2012; Reines, Greene \& Geha 2013; Baldassare et al. 2015; Reines \& Volonteri 2015; Chilingarian et al. 2018; Woo et al. 2018, 2019; Baldassare et al. 2020), (2) the accretion signatures of narrowline emission (e.g. Moran et al. 2014) and coronal emission in the mid-infrared (MIR; Satyapal et al. 2009), (3) tidal-disruption events (TDEs; e.g. Maksym et al. 2013; Stone, Küpper \& Ostriker 2017), 
(4) hard X-ray emission (e.g. Gallo et al. 2008; Desroches, Greene \& Ho 2009; Gallo et al. 2010; Miller et al. 2015; She, Ho \& Feng $2017 b$, a), (5) detection of other emission lines, such as $\mathrm{Br} \gamma$, that might be associated with X-ray radiation from the accreting region (Osterbrock 1989; Panessa et al. 2006; Cresci et al. 2010; Reines et al. 2011; Nguyen et al. 2014), (6) the dynamics of accretion discs containing megamasers (Miyoshi et al. 1995; Lo 2005; Kuo et al. 2011; van den Bosch et al. 2016), and (7) the dynamics of stars and warm/ionized gas (Verolme et al. 2002; Valluri et al. 2005; Neumayer et al. 2007; Seth et al. 2010; van den Bosch \& de Zeeuw 2010; den Brok et al. 2015; Nguyen 2017; Nguyen et al. 2017, 2018, 2019; Thater et al. 2017, 2019; Krajnović et al. 2018) and dynamically CMC (Combes et al. 2019; Davis et al. 2020; this work) in lowmass galaxies $\left(5 \times 10^{8}<M_{\star} \lesssim 10^{10} \mathrm{M}_{\odot}\right)$ and ultracompact dwarfs (UCDs; $1 \times 10^{7}<M_{\star} \leq 5 \times 10^{8} \mathrm{M}_{\odot}$; Seth et al. 2014; Ahn et al. 2017; Afanasiev et al. 2018; Ahn et al. 2018; Voggel et al. 2018). The importance of the $\lesssim 10^{6} \mathrm{M}_{\odot} \mathrm{BH}$ population is discussed in detail in Nguyen et al. (2017), Nguyen et al. (2018), Nguyen et al. (2019).

We started the project 'Measuring Black Holes in Below Milky Way-mass $\left(\mathbf{M}^{\star}\right)$ galaxies' (MBHBM ${ }^{\star}$ Project; Nguyen 2019; Nguyen et al. 2020) to gather a large sample of gas-rich galaxies with reliably measured SMBH masses in the regime $M_{\star} \lesssim 5 \times 10^{10} \mathrm{M}_{\odot}$ (e.g. Baldry et al. 2012; Cautun et al. 2020) and $\sigma_{\star}<120 \mathrm{~km} \mathrm{~s}^{-1}$, where stellar kinematics of sufficiently high spatial and spectral resolutions are hard to obtain. Instead, we use CMC tracers observed with Atacama Large Millimeter/submillimeter Array (ALMA) to measure their central kinematics and thus dark central masses, that are likely BHs. The outcomes of this project will reveal the demographics of the $\lesssim 10^{5}-10^{7} \mathrm{M}_{\odot}$ BHs (i.e. the regime with currently sparse or limited observations) and provide enough measurements to accurately constrain the scatters and slopes of BH-galaxy scaling relations at the low-mass end. The molecular gas method is also the most reliable method to precisely measure the occupation fraction $\left(f_{\text {occ }}\right)$ of central BHs among low-mass galaxies, an important parameter to constrain the possible $\mathrm{BH}$ seed formation mechanisms in the early Universe (e.g. Greene 2012; Reines \& Volonteri 2015; Greene, Strader \& Ho 2020; Neumayer, Seth \& Boeker 2020), either the direct collapse of gas clouds $\left(f_{\text {occ }}<60\right.$ per cent; e.g. Lodato \& Natarajan 2006; Gallo et al. 2008; Bonoli, Mayer \& Callegari 2014; Miller et al. 2015) or the death of the first stars $\left(f_{\text {occ }}>60\right.$ per cent; e.g. Volonteri, Lodato \& Natarajan 2008; van Wassenhove et al. 2010; Volonteri 2010, 2012; Volonteri \& Bellovary 2012; Reines \& Comastri 2016; Haemmerlé et al. 2020; Inayoshi, Visbal \& Haiman 2020; ).

Many recent works have used molecular gas tracers to weigh central BHs dynamically, proving this method can be applied to a variety of galaxy types and masses. The method was first pioneered with Combined Array for Research in Millimetre-wave Astronomy (CARMA) observations of the galaxy NGC 4526 (Davis et al. 2013) and has

now been applied to both active and non-active as well as earlytype (ETGs; Barth et al. 2016a,b; Davis et al. 2013, 2017, 2018; Onishi et al. 2017; Boizelle et al. 2019, 2021; Combes et al. 2019; Nagai et al. 2019; North et al. 2019; Smith et al. 2019, 2021a; Thater 2019; Cohn et al. 2021; Thater et al., in preparation; Nguyen et al., in preparation) and late-type (LTGs; Onishi et al. 2015; Combes et al. 2019; Nguyen et al. 2020, 2021; Boizelle et al. 2021) galaxies with ALMA and CARMA. The SMBH masses have in fact now been shown to correlate with the molecular gas line widths (Smith et al. 2021b) and there are thousands of potential targets (Davis 2014). This method has also allowed some of the most accurate $M_{\mathrm{BH}}$ measurements to date, in the radio galaxy NGC $0383\left(M_{\mathrm{BH}}=\right.$ $(4.2 \pm 0.2) \times 10^{9} \mathrm{M}_{\odot} ;$ North et al. 2019) and the non-active elliptical galaxy NGC $3258\left(M_{\mathrm{BH}}=(2.249 \pm 0.004) \times 10^{9} \mathrm{M}_{\odot}\right.$; Boizelle et al 2019). These measurements rival the best megamaser measurements, up to now the 'gold standard' of extragalactic $M_{\mathrm{BH}}$ measurements. The molecular gas method also extends accurate measurements towards the regime of $\lesssim 10^{6} \mathrm{M}_{\odot}$ BHs, for example in NGC 404 with $M_{\mathrm{BH}}=5_{-2}^{+1} \times 10^{5} \mathrm{M}_{\odot}$ (Davis et al. 2020) and now NGC 3593 in this work. All of these works prove that the cold-gas dynamical method combined with ALMA observations at high angular resolutions can now be effective over a range of $\mathrm{BH}$ masses covering six orders of magnitude $\left(10^{5}-10^{10} \mathrm{M}_{\odot}\right)$.

This paper is the second of a series from the $\mathrm{MBHBM}^{\star}$ Project (Nguyen 2019), following the first measurement in the nearby double-bar LTG NGC $3504\left(M_{\mathrm{BH}}=1.6_{-0.4}^{+0.6} \times 10^{7} \mathrm{M}_{\odot}\right.$; Nguyen et al. 2020). The paper is organized into eight sections. The properties of the target galaxy NGC 3593 are presented in Section 2. In Section 3, we present Hubble Space Telescope (HST) images of the galaxy and ALMA observations of the nuclear ${ }^{12} \mathrm{CO}(2-1)$ emission, discussing in detail our data reduction and analysis. We describe the Kinematic Molecular Simulation (KinMS; Davis et al. 2013) model that we use to constrain the mass profile of the galaxy in Section 4 and the inferred central $M_{\mathrm{BH}}$ in Section 5. We also determine the masses and sizes of the nuclear star cluster (NSC) and massive core of cold molecular gas (CMC) in Section 6. We further discuss our results in Section 7 and conclude in Section 8.

Throughout this work, we (1) quote all quantities using a foreground extinction correction $A_{V}=0.053$ mag (Schlafly \& Finkbeiner 2011) and the Cardelli, Clayton \& Mathis (1989) interstellar extinction law and (2) adopt a Tully-Fisher (TF) distance ${ }^{2}$ to NGC 3593 of $7 \pm 2$ Mpc (Wiklind \& Henkel 1992), with the uncertainty based on the spread of TF distances in the National Aeronautics and Space Administration (NASA) Infrared Processing and Analysis Center (IPAC) Extragalactic Database $\left(\mathrm{NED}^{3}\right)$, yielding a physical scale of $\approx 35 \mathrm{pc} \operatorname{arcsec}^{-1}$ assuming a current Hubble constant $H_{0}=$ $70.3 \pm 1.6 \mathrm{~km} \mathrm{~s}^{-1} \mathrm{Mpc}^{-1}$, matter density (with respect to the critical mass density) $\Omega_{\mathrm{m}, 0}=0.277 \pm 0.019$ and dark energy density (with respect to the critical mass density) $\Omega_{\Lambda, 0}=0.723 \pm 0.019$ from the Wilkinson Microwave Anisotropy Probe (WMAP; Moura-Santos et al. 2016; Verschuur \& Schmelz 2016; Calabrese et al. 2017) and PLANCK Collaboration (Planck Collaboration XVI 2014). All the maps presented in this paper are plotted with north up and east to the left. Tables and Figures labelled with numbers only appear in order in the main text, while those labelled with both letters and numbers appear in the corresponding appendices.

\section{NGC 3593}

We summarize the known properties of NGC 3593 in Table 1 and further discuss these properties in detail in this section.

NGC 3593 (UGC 6272) is classified as a lenticular galaxy (Buta, Corwin \& Odewahn 2007), with a morphological classification of $\mathrm{SA}(\mathrm{s}) 0 / \mathrm{a}$ or numerical Hubble type $T_{\text {Hubble }}=-0.4 \pm 0.9$ (NED) and it is a member of the Leo Group (Stierwalt et al. 2009). It has a dust disc obscuring the galaxy central regions to the north of the major axis (Sandage \& Bedke 1994).

\footnotetext{
${ }^{2}$ The choice of distance $D$ does not influence our conclusions but merely sets the scale of our models in physical units. In particular, lengths and dynamically derived masses such as $M_{\mathrm{BH}}$ scale as $D$, luminosity-derived masses scale as $D^{2}$, and mass-to-light ratios scale as $D^{-1}$.

${ }^{3}$ https://ned.ipac.caltech.edu/
} 
Table 1. Properties of NGC 3593.

\begin{tabular}{|c|c|c|}
\hline Parameter (units) & Value & References \\
\hline Morphology & $\mathrm{SA}(\mathrm{s}) 0 / \mathrm{a}$ & $(1,2)$ \\
\hline RA (J2000) & $11^{\mathrm{h}} 14^{\mathrm{m}} 37^{\mathrm{s}} .1$ & (3) \\
\hline Dec. (J2000) & $+12^{\circ} 49^{\prime} 05^{\prime \prime} .6$ & (3) \\
\hline Position angle $\left({ }^{\circ}\right)$ & 90 & (4) \\
\hline Inclination angle $\left(^{\circ}\right)$ & 67 & (5) \\
\hline Systemic velocity $\left(\mathrm{km} \mathrm{s}^{-1}\right)$ & 629 & (3) \\
\hline Distance (Mpc) & 7 & (6) \\
\hline Linear scale $\left(\mathrm{pc} \operatorname{arcsec}^{-1}\right)$ & 35 & $(7,8)$ \\
\hline $\log \left(L_{\left.2-10 \mathrm{keV} / \mathrm{erg} \mathrm{s}^{-1}\right)}\right.$ & $<38.23$ & (9) \\
\hline $\log \left(L_{0.3-8 \mathrm{keV}} / \mathrm{erg} \mathrm{s}^{-1}\right)$ & 38.33 & (10) \\
\hline Total stellar mass $\left(\mathrm{M}_{\odot}\right)$ & $1.5 \times 10^{10}$ & (11) \\
\hline Total H I mass $\left(\mathrm{M}_{\odot}\right)$ & $1.3 \times 10^{8}$ & (12) \\
\hline Total dust mass $\left(\mathrm{M}_{\odot}\right)$ & $1.0 \times 10^{6}$ & (13) \\
\hline Dust temperature (K) & 40 & (13) \\
\hline Stellar velocity dispersion $\left(\mathrm{km} \mathrm{s}^{-1}\right)$ & 60 & (11) \\
\hline$\langle S F R\rangle_{\mathrm{CND}}\left(\mathrm{M}_{\odot} \mathrm{yr}^{-1}\right)$ & 3 & (14) \\
\hline Stellar properties & (age, $[\mathrm{Z} / \mathrm{H}])$ & \\
\hline Main component & $(3.6 \mathrm{Gyr},-0.04)$ & $(15)$ \\
\hline Secondary component & $(2.0 \mathrm{Gyr},-0.15)$ & (15) \\
\hline \multicolumn{3}{|l|}{ NSC properties } \\
\hline Effective radius (pc) & $5.50 \pm 0.23$ & (16) \\
\hline Sérsic index & $1.40 \pm 0.14$ & (16) \\
\hline Mass $\left(\mathrm{M}_{\odot}\right)$ & $1.58 \times 10^{8}$ & (16) \\
\hline$L_{I}\left(\mathrm{~L}_{\odot}\right)$ & $2.89 \times 10^{7}$ & (16) \\
\hline
\end{tabular}

Notes. (1) Sandage \& Tammann (1981); (2) de Vaucouleurs et al. (1991); (3) García-Burillo et al. (2000); (4) Sandage \& Tammann (1981); (5) Rubin et al. (1985); (6) Wiklind \& Henkel (1992); (7) Moura-Santos et al. (2016); (8) Planck Collaboration XVI (2014); (9) She et al. (2017b); (10) Zhang et al. (2009); (11) Bertola et al. (1996); (12) Pogge \& Eskridge (1993); (13) Fich (1993); (14) Ho, Filippenko \& Sargent (1997); (15) Coccato et al. (2013); and (16) Pechetti et al. (2020).

NGC 3593 is known to contain two distinct stellar populations that are rotating in opposite directions (Bertola et al. 1996; Corsini et al. 1998; García-Burillo et al. 2000; Coccato et al. 2013). The main stellar component is slightly older and more metal-rich (luminosityweighted age of $3.6 \pm 0.6 \mathrm{Gyr}$ and metallicity $[\mathrm{Z} / \mathrm{H}]=-0.04 \pm 0.03$ ) and is rotating slightly slower (rotation velocity around the nucleus of $\approx 100 \mathrm{~km} \mathrm{~s}^{-1}$ ), while the secondary (counterrotating) stellar component is slightly younger and more metal-poor (age $2.0 \pm 0.5 \mathrm{Gyr}$ and metallicity $[\mathrm{Z} / \mathrm{H}]=-0.15 \pm 0.07)$ and is rotating slightly faster $\left(\approx 120 \mathrm{~km} \mathrm{~s}^{-1}\right.$; Coccato et al. 2013). Such a configuration can arise from the merger of a dwarf galaxy (Balcells \& González 1998; Jesseit et al. 2007; Bois et al. 2011; Eliche-Moral et al. 2011), although an alternative explanation is that the galaxy nucleus accreted gas on retrograde orbits from an external source, that then underwent star formation. The stellar velocity dispersions of these two stellar components are in the range $30-80 \mathrm{~km} \mathrm{~s}^{-1}$, while Bertola et al. (1996) measured $\sigma_{\star} \approx 60 \mathrm{~km} \mathrm{~s}^{-1}$ in the galaxy centre, suggesting an $\approx 1.7_{-1.1}^{+3.2} \times 10^{6} \mathrm{M}_{\odot}$ central $\mathrm{BH}$ based on the Kormendy \& Ho (2013) $M_{\mathrm{BH}}-\sigma_{\star}$ relation for massive ETGs.

The nucleus of NGC 3593 is red $(F 555 \mathrm{~W}-F 814 \mathrm{~W} \approx 2.5 \mathrm{mag})$, due to the presence of dust and a luminous and massive NSC ( $I$ band luminosity $L_{I, \mathrm{NSC}}=2.89 \times 10^{7} \mathrm{~L}_{\odot}$ and stellar mass $M_{\mathrm{NSC}}=$ $\left.1.58 \times 10^{8} \mathrm{M}_{\odot}\right)$, that has a Sérsic index $n_{\mathrm{NSC}}=1.4 \pm 0.14$ and an effective (half-light) radius $r_{\mathrm{NSC}, \mathrm{e}}=5.50 \pm 0.23 \mathrm{pc}$ (Pechetti et al. 2020; although see our improved measurements in Sections 6.1).

Bertola et al. (1996) also performed a photometric decomposition using an $r$-band stellar surface-brightness map obtained from the spectral decomposition of European Southern Observatory (ESO) 1.5-m spectroscopic telescope data, and found two stellar discs with the same radially constant ellipticity $(\epsilon=0.55)$ and position angle $\left(\mathrm{PA}=90^{\circ}\right)$. The two stellar discs can be parameterised by infinitely thin exponential discs with different scale lengths $(h)$, central surface brightnesses $(\mu)$ and thus total stellar masses $\left(M_{\star}\right): h_{1}=40 \operatorname{arcsec}$ $(1.4 \mathrm{kpc}), \mu_{1}=19.9 \mathrm{mag} \operatorname{arcsec}^{-2}$ and $M_{\star, 1}=1.2 \times 10^{10} \mathrm{M}_{\odot}$, and $h_{2}=10 \operatorname{arcsec}(350 \mathrm{pc}), \mu_{2}=18.5 \mathrm{mag} \operatorname{arcsec}^{-2}$ and $M_{\star, 2}=$ $2.7 \times 10^{9} \mathrm{M}_{\odot}$. The masses were obtained by fitting the ionized gas rotation curve, allowing the mass-to-light ratios of the two discs to vary independently. NGC 3593 has a total stellar mass of $M_{\star} \approx$ $1.5 \times 10^{10} \mathrm{M}_{\odot}$ (Bertola et al. 1996) and thus can be classified as a sub- $M^{\star}$ galaxy (Baldry et al. 2012).

Narrow-band $\mathrm{H} \alpha+[\mathrm{NII}](\lambda \lambda$ 654.80, $658.34 \mathrm{~nm})$ and [S II] $(\lambda \lambda 671.65,673.08 \mathrm{~nm})$ observations reveal a ring of ionized gas in the circumnuclear region of NGC 3593, that extends to a radius $r \approx 17 \operatorname{arcsec}$ (or $\approx 595 \mathrm{pc}$; Corsini et al. 1998). The ionized gas kinematics show that this material rotates in the same sense as the secondary/counterrotating stellar component and has a velocity dispersion $\lesssim 30 \mathrm{~km} \mathrm{~s}^{-1}$.

Using Institut de Radioastronomie Millimétrique (IRAM) Plateau de Bure Interferometer (PdBI) ${ }^{12} \mathrm{CO}(1-0)$ observations at an angular resolution of $4 \times 3$ arcsec, García-Burillo et al. (2000) found that NGC 3593 has an inner disc of molecular gas extending to $r \approx$ $35 \operatorname{arcsec}$ ( or $\approx 1.2 \mathrm{kpc}$ ), counterrotating at all radii with respect to the most massive/primary stellar disc. Half of the ${ }^{12} \mathrm{CO}(1-0)$ emission (and hence half of the associated mass) arises from an elongated circumnuclear disc (CND) within a region of radius $r \approx$ $10 \operatorname{arcsec}$ (or $\approx 350 \mathrm{pc}$ ), with an outer ring-like structure and a two-arm/bi-symmetric spiral pattern within it. This CND connects to outer gas reservoirs containing the remaining half of the ${ }^{12} \mathrm{CO}(1-0)$ emission (and thus of its associated mass) and extending out to $r \approx$ 35 arcsec, which allows the gas to flow to the northern half of the disc (García-Burillo et al. 2000). Pogge \& Eskridge (1993) report a total neutral hydrogen $(\mathrm{HI})$ gas mass of $\approx 1.3 \times 10^{8} \mathrm{M}_{\odot}$, which is a compilation from the RC3 catalogue (Corwin Harold, Buta \& de Vaucouleurs 1994).

The nucleus of NGC 3593 is classified as an H II star-forming nucleus (Hunter et al. 1989), with an upper limit on the total star formation rate $(\mathrm{SFR})$ of the $\mathrm{CND}$ alone $\left(\mathrm{SFR}_{\mathrm{CND}} \lesssim 3 \mathrm{M}_{\odot} \mathrm{yr}^{-1}\right.$; Ho et al. 1997). This upper limit is due to $\mathrm{H} \beta$ emission being almost absent from the centre of the galaxy. A similar behaviour is seen in other high-resolution observations of star formation tracers such as $\mathrm{H} \alpha$ and $\mathrm{Pa} \alpha$ (García-Burillo et al. 2000), fuelling the second/counterrotating stellar disc. Optical and near-infrared (NIR) recombination lines suggest a $V$-band extinction $A_{V} \approx 1 \mathrm{mag}$ in the CND, while the $\mathrm{CO}$ and $100-\mu \mathrm{m}$ fluxes suggest $A_{V}>5 \mathrm{mag}$ (García-Burillo et al. 2000).

The total mass and temperature of the dust of NGC 3593 were estimated to be $T_{\text {dust }} \approx 40 \mathrm{~K}$ and $M_{\text {dust }} \approx 10^{6} \mathrm{M}_{\odot}$ using observations at $1.1 \mathrm{~mm}, 800$ and $450 \mu \mathrm{m}$ (Fich 1993), yielding a gas-to-dust mass ratio of $\approx 300$. This ratio is two times higher than the canonical value of $\approx 150$ derived from $\mathrm{CO}$ lines associated with high extinction regions and widely used for the Galaxy (Spitzer 1978; Hildebrand 1983; Draine \& Lee 1984).

The X-ray detection in the nucleus of NGC 3593 with Chandra is debated. Martínez-García et al. (2017) and She et al. (2017a) found no X-ray emission, and She et al. (2017b) reported an upper limit on the X-ray luminosity of $\log \left(L_{2-10 \mathrm{keV}} / \mathrm{erg} \mathrm{s}^{-1}\right)<38.23$. On the other hand, Zhang et al. (2009) do report an X-ray detection and a nuclear luminosity of $\log \left(L_{0.3-8 \mathrm{kev}} / \mathrm{erg} \mathrm{s}^{-1}\right)=38.33$. The reason behind these different conclusions is as yet unknown, but it may be the different detection criteria adopted. In any case, the SMBH of NGC 3593, as inferred from $M_{\mathrm{BH}}-\sigma_{\star}$ correlations, must be accreting 
Table 2. HST/WFPC2 data.

\begin{tabular}{lcccc}
\hline Filter & $\begin{array}{c}\text { Pixel scale }^{a} \\
\left(\text { arcsec pixel }^{-1}\right) \\
(2)\end{array}$ & $\begin{array}{c}\text { Exposure time } \\
(\mathrm{s})\end{array}$ & $\begin{array}{c}\text { Zero point } \\
(\mathrm{mag})\end{array}$ & $\begin{array}{c}A_{\lambda}^{c} \\
(\mathrm{mag}) \\
(1)\end{array}$ \\
\hline F450W & 0.0455 & $2 \times 200$ & 24.11 & 0.066 \\
F814W & 0.0455 & $2 \times 130$ & 23.76 & 0.030 \\
\hline
\end{tabular}

$\overline{{ }^{a} \text { Holtzman et al. (1995). }{ }^{b} \text { Vega System. }{ }^{c} \text { Foreground extinction correction }}$ assuming a Milky-Way interstellar extinction law from ultraviolet to NIR (Cardelli et al. 1989; Schlafly \& Finkbeiner 2011).

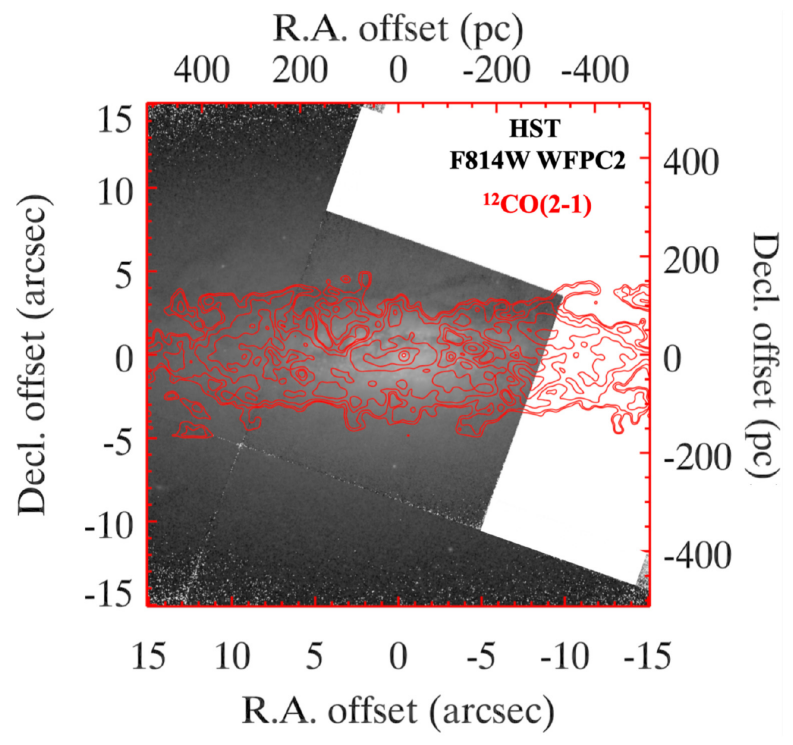

Figure 1. HST/WFPC2 F814W image of NGC 3593 with a field-of-view of $30 \times 30 \operatorname{arcsec}^{2}\left(1.05 \times 1.05 \mathrm{kpc}^{2}\right)$, overlaid with the ${ }^{12} \mathrm{CO}(2-1)$ integrated intensity contours from our ALMA observations. The HST image is in an arbitrary logarithmic scale. Grey dust lanes are clearly visible on the northern side of the nucleus.

at an extremely low rate, $\dot{M}_{\mathrm{BH}} / M_{\mathrm{BH}} \lesssim 10^{-7}$ of the Eddington limit. Thus, there is no evidence for an active galactic nucleus (AGN) in NGC 3593.

\section{DATA AND DATA REDUCTION}

\subsection{HST images}

We use HST Wide-Field Planetary Camera 2 (WFPC2) images in the F450W and $F 814 W$ bands taken on 2007 November 20 (GO-11128, PI: Fisher) to create a central stellar mass model of NGC 3593 (see Section 4.3), that will be used as an input to our dynamical models in Section 5. More details of these images are listed in Table 2.

The photometric centre of the galaxy in the HST/WFPC2 images is offset by $\left(-0.13,-0^{\prime \prime} 11\right)$ with respect to the photometric and kinematic centre of NGC 3593, as determined from our own high-resolution ${ }^{12} \mathrm{CO}(2-1)$ data and discussed in more details in Section 3.4. This is within the positional uncertainty of the HST images, so we align the $H S T$ images to this ${ }^{12} \mathrm{CO}(2-1)$ centre to correct for the astrometric mismatch, and show the (offset) F814W image overlaid with the ${ }^{12} \mathrm{CO}(2-1)$ isointensity contours in Fig. 1.

We used Tiny Tim point spread functions (PSFs; Krist 1995; Krist, Hook \& Stoehr 2011) of the WFPC2 F450W and F814W images to create (1) a $F 555 \mathrm{~W}-F 814 \mathrm{~W}$ colour map and (2) a multi-
Gaussian expansion (MGE; Emsellem, Monnet \& Bacon 1994; Cappellari 2002) stellar light model in Section 4.2.

\section{$3.2{ }^{12} \mathrm{CO}(2-1)$ ALMA observations}

Our ALMA observations of NGC 3593 were carried out on 2018 September 23 (PID: 2017.1.00964.S, PI: Nguyen, Dieu). The ${ }^{12} \mathrm{CO}(2-1)$ emission line was observed for a total of $90 \mathrm{~min}$ (54 min on source) using 49 ALMA 12-m antennae in the C43-5 configuration (baseline range $15-1400 \mathrm{~m}$ ), resulting in a maximum recoverable scale (MRS) of $\approx 30$ arcsec in diameter and a synthesised beam full width at half-maximum $(\mathrm{FWHM})$ of $0.33 \times 0.29\left(11.6 \times 10.2 \mathrm{pc}^{2}\right)$ oriented at $\mathrm{PA}=35^{\circ}$. The correlator was set up using four spectral windows, including one window covering the ${ }^{12} \mathrm{CO}(2-1)$ line in frequency division mode (FDM; $1875 \mathrm{MHz}$ bandwidth with $1.13 \mathrm{MHz}$ or $\approx 1.5 \mathrm{~km} \mathrm{~s}^{-1}$ channels) and three windows to probe continuum emission in time division mode (TDM; $2 \mathrm{GHz}$ bandwidth with $31.25 \mathrm{MHz}$ or $\approx 40.7 \mathrm{~km} \mathrm{~s}^{-1}$ channels). The raw ALMA data were calibrated by ALMA Regional Center staff using the standard ALMA pipeline. Flux and bandpass calibrations were carried out using the quasars J1037-2934, J0854+2006 and J1118+1234, while the atmospheric phase offsets were determined using the quasar $\mathrm{J} 1103+1158$.

No continuum emission is detected across the primary beam of $25^{\prime \prime} .3$ diameter, so we report an upper limit on the continuum emission of $\approx 30 \mu \mathrm{Jy}$ beam $^{-1}$, resulting from a summation of all line-free channels across all four spectral windows. Also as a result, we created a three-dimensional (3D; RA, Dec., velocity) datacube directly from the calibrated measurement set without continuum subtraction using the clean task of the Common Astronomy Software Applications (CASA; McMullin et al. 2007) package version 5.1.1, following the same successful strategy employed in our previous studies (e.g. Davis et al. 2017; Nguyen et al. 2020). Specifically, we created the ALMA ${ }^{12} \mathrm{CO}(2-1)$ datacube of NGC 3593 with a pixel size of $0^{\prime \prime} 1$, a binned channel width of $10 \mathrm{~km} \mathrm{~s}^{-1}$ (this is several times the raw channel width of $\approx 1.5 \mathrm{~km} \mathrm{~s}^{-1}$, such that the channels are effectively independent) and Briggs weighting with a robust parameter of 0.5 . For the clean task, we used the interactive masking mode to further reduce the sidelobes of the data, estimating the root mean square (RMS) noise in a few channels of the residual cube and setting $3 \times$ RMS as the cleaning threshold in regions of source emission in dirty channels. We performed primary beam correction after cleaning. Our final fully calibrated and cleaned ${ }^{12} \mathrm{CO}(2-1)$ datacube has an $\mathrm{RMS}$ noise of $\approx 1 \mathrm{mJy}^{\text {beam }}{ }^{-1}$ per $10 \mathrm{~km} \mathrm{~s}^{-1}$ binned channel and emission is detected from $\approx 500$ to $\approx 750 \mathrm{~km} \mathrm{~s}^{-1}$ with a mean (systemic) velocity of $629 \mathrm{~km} \mathrm{~s}^{-1}$.

\section{$3.3{ }^{12} \mathrm{CO}(2-1)$ moment maps}

For example, Fig. B1 in Appendix B shows the ${ }^{12} \mathrm{CO}(2-1)$ integrated intensity (moment 0 ), intensity-weighted mean line-of-sight (LOS) velocity (moment 1 ) and intensity-weighted LOS velocity dispersion (moment 2) maps of the CND region, roughly matching the 12-m antennae primary beam. We created these maps using the momentmasking technique (Dame, Hartmann \& Thaddeus 2001; Dame 2011). The mask was thus created by first spatially smoothing each channel by a factor of $\alpha$ by convolving each channel by a Gaussian of FWHM $\alpha$ times the FWHM of the synthesized beam, where we varied $\alpha$ and gauged the spatial and velocity coherence of the signal. Spatial smoothing increases the sensitivity while decreasing the angular resolution, helping to expunge noise peaks. Second, we performed ' $\beta \times \sigma$-clipping', where $\beta$ is a positive factor and $\sigma$ the 
RMS noise. Here, at any given position, all channels with intensities below $\beta \sigma$ were set to zero. We note that this mask created from the smoothed cube is only used to identify and mask out emission-free regions of the original cube; the latter is used to create the moment maps at full spatial and velocity resolutions. We experimented with appropriate choices of the smoothing and masking parameters to obtain the best moment maps, finally adopting $\alpha=3$ and $\beta=0.75$.

The ${ }^{12} \mathrm{CO}(2-1)$ emission is significant within an $\approx 30 \times 10 \operatorname{arcsec}^{2}$ rectangular central region roughly corresponding to the CND (see Panel A of Fig. B1 in Appendix B) and peaking at (RA, Dec.) = $\left(11^{\mathrm{h}} 14^{\mathrm{m}} 37^{\mathrm{s}} .1,+12^{\circ} 49^{\prime} 05^{\prime \prime} \cdot 6\right)$, that is identified as the galaxy centre (see Section 3.4). Interestingly, there is a distinct massive core of cold molecular gas (CMC; radius $r \lesssim 0$ '.5) embedded within the dense CND ( $r \lesssim 10$ arcsec), itself surrounded by a more diffuse and extended gas disc $(10 \lesssim r \lesssim 35$ arcscec). The CND has an outer ring-like structure (radius $\approx 10$ arcsec) and a two-arm/bi-symmetric spiral pattern within it, apparently extending down to the CMC. This pattern seems to match the ridge of high-velocity dispersion regions in the moment 2 map.

The intensity-weighted mean LOS velocity map in panel $\mathrm{C}$ of Fig. B1 in Appendix B confirms that the central molecular gas is consistent with a rotating disc, with a total velocity width $\Delta V \approx$ $250 \mathrm{~km} \mathrm{~s}^{-1}$. This rotation is consistent with both that of the counterrotating stellar component measured using Very Large Telescope (VLT) Visible Multi-object Spectrograph (VIMOS) integral-field observations (Coccato et al. 2013) and that of the counterrotating ionized-gas component measured using ESO's 1.5-m spectroscopic telescope (Bertola et al. 1996). The rotational velocities of the molecular gas are however higher than those of the primary stellar component $\left(\Delta V \approx 200 \mathrm{~km} \mathrm{~s}^{-1}\right)$.

The intensity-weighted LOS velocity dispersion map in Panel D of Fig. B1 in Appendix B is quite flat, with a roughly constant dispersion $\sigma \approx 10 \mathrm{~km} \mathrm{~s}^{-1}$, except for a few regions of higher velocity dispersion $\left(25-33 \mathrm{~km} \mathrm{~s}^{-1}\right.$ ) coincident with bright emission clumps. The velocity dispersion of the molecular gas is consistent with that of the ionized gas $\left(\$ 30 \mathrm{~km} \mathrm{~s}^{-1}\right.$; Bertola et al. 1996). The high-velocity dispersion at the very centre is consistent with the existence of the CMC.

For example, Fig. B2 in Appendix B shows the ${ }^{12} \mathrm{CO}(2-1)$ integrated spectrum of NGC 3593, with the classic double-horn shape of a rotating disc. We also plot the position-velocity diagram (PVD) extracted from a cut along the kinematic major axis of the disc $(\mathrm{PA}=$ $\left.90^{\circ}\right)$ in Fig. B3 in Appendix B. There is a sharp increase of the rotation towards the galaxy centre ( $r \lesssim 1 \operatorname{arcsec}$ or $35 \mathrm{pc}$ ). We later interpret this rotation as being caused by the massive cores (NSC and CMC) and/or an SMBH at the centre of the galaxy.

In Fig. 2 , we show a zoom (inner $\approx 4 \times 4 \operatorname{arcsec}^{2}$ or $\approx 140 \times$ $140 \mathrm{pc}^{2}$ ) of the integrated intensity map (Panel A), intensity-weighted mean LOS velocity map (Panel B), intensity-weighted LOS velocity dispersion map (Panel C) and PVD extracted along the kinematic major axis of the CND (Panel D). Specifically, Panel A illustrates the detailed ${ }^{12} \mathrm{CO}(2-1)$ morphology of the $\mathrm{CND}$, with the bright $\mathrm{CMC}$ at its centre. Panel B shows the central molecular gas kinematics under the influence of the compact central massive objects (i.e. NSC, CMC, and SMBH) at radii $r \lesssim 1$ arcsec, and as seen in the PVD in Panel D. Some non-circular motions may be present in the blueshifted half of the velocity map away from the kinematic major axis.

\subsection{Galaxy centre}

As can be seen from the different panels of Fig. 2, the photometric (integrated intensity peak or CMC) and kinematic centre of our highresolution ${ }^{12} \mathrm{CO}(2-1)$ data are consistent with each other. Within the stated uncertainties, these centres also agree with the kinematic centre derived by García-Burillo et al. (2000) from lower-resolution ${ }^{12} \mathrm{CO}(1-0)$ data, and with the optical photometric centre derived from Sloan Digital Sky Survey (SDSS) data release 14 (DR14) data (Abolfathi et al. 2018). We therefore adopt this centre $\left(\mathrm{RA}=11^{\mathrm{h}} 14^{\mathrm{m}} 37^{\mathrm{s}} .1\right.$, Dec. $=+12^{\circ} 49^{\prime} 05^{\prime \prime} .6, V_{\mathrm{sys}}=629 \mathrm{~km} \mathrm{~s}^{-1}$ ) as the centre of NGC 3593 .

\section{MASS MODEL}

In this section, we first use our ${ }^{12} \mathrm{CO}(2-1)$ kinematics (see Section 3.2) and a dynamical model to constrain the outer part $(4 \leq r \leqslant 30 \operatorname{arcsec}$ or $140 \leq r \lesssim 1050 \mathrm{pc}$ ) of our galaxy mass model (Section 4.2). Second, we use the HST imaging data (see Section 3.1) to constrain the inner part $(r<4 \operatorname{arcsec}$ or $r<140 \mathrm{pc}$ ) of our galaxy mass model (Section 4.3). The combination of the inner and outer mass model yields a complete mass model of NGC 3593 , from the centre to a radius of $30 \operatorname{arcsec}$ (Section 4.4), a key ingredient to estimate the central $M_{\mathrm{BH}}$ through dynamical modelling.

\subsection{KinMS model}

The KinMS tool, we use for dynamical modelling comprises two main elements. First, for a given set of model parameters, it creates a simulated data cube for comparison to observations. Second, it explores parameter-space in an efficient manner to identify the bestfitting model.

To simulate a data cube, KinMS adopts a parametric function (specified with some free parameters) describing the distribution of the (massless) kinematic tracer [here ${ }^{12} \mathrm{CO}(2-1)$ emission]. Here, we also assume the tracer moves on circular orbits governed by a circular velocity curve, calculated from the mge_circular_velocity procedure within the Interactive Data Language (IDL) Jeans Anisotropic Modelling ( $\mathrm{JAM}^{4}$; Cappellari 2008) package, that itself uses as an input an (axisymmetric) mass model specified via MGE parametrization (see Sections 4.2 and 4.3), that can include any number of mass components (here stars, gas, dust and the putative SMBH; see Davis et al. 2013).

The KinMS tool simulates the whole cube, then compares it to the data via a likelihood function (Davis et al. 2017, 2018; Onishi et al. 2017; North et al. 2019; Smith et al. 2019, 2021b; Nguyen et al. 2020, 2021; Thater et al. 2020). During the fit, the model, walks through parameter space using a Markov chain Monte Carlo (MCMC) method controlled by the emcee algorithm (Foreman-Mackey et al. 2013) and an affine-invariant ensemble sampler (Goodman \& Weare 2010) in a Bayesian framework. At each step, the relative likelihood is calculated and used to determine the next move through parameter space. The best-fitting model is then determined from the full posterior distribution. In practice, this is all achieved by using the python code KINMSpy_MCMC. ${ }^{5}$

\subsection{Outer mass model}

We model the outer part of the galaxy mass distribution $(4 \leq r \lessgtr 30$ arcsec or $140 \leq r \lesssim 1,050 \mathrm{pc}$ ) with two mass components described below: a stellar one with free mass normalization and an interstellar medium (ISM) one that is fixed.

For the stellar component, we first average the mass surfacedensity profiles of the primary and secondary/counterrotating stellar

\footnotetext{
${ }^{4}$ https://purl.org/cappellari/software

${ }^{5}$ https://github.com/TimothyADavis/KinMS_MCMC
} 

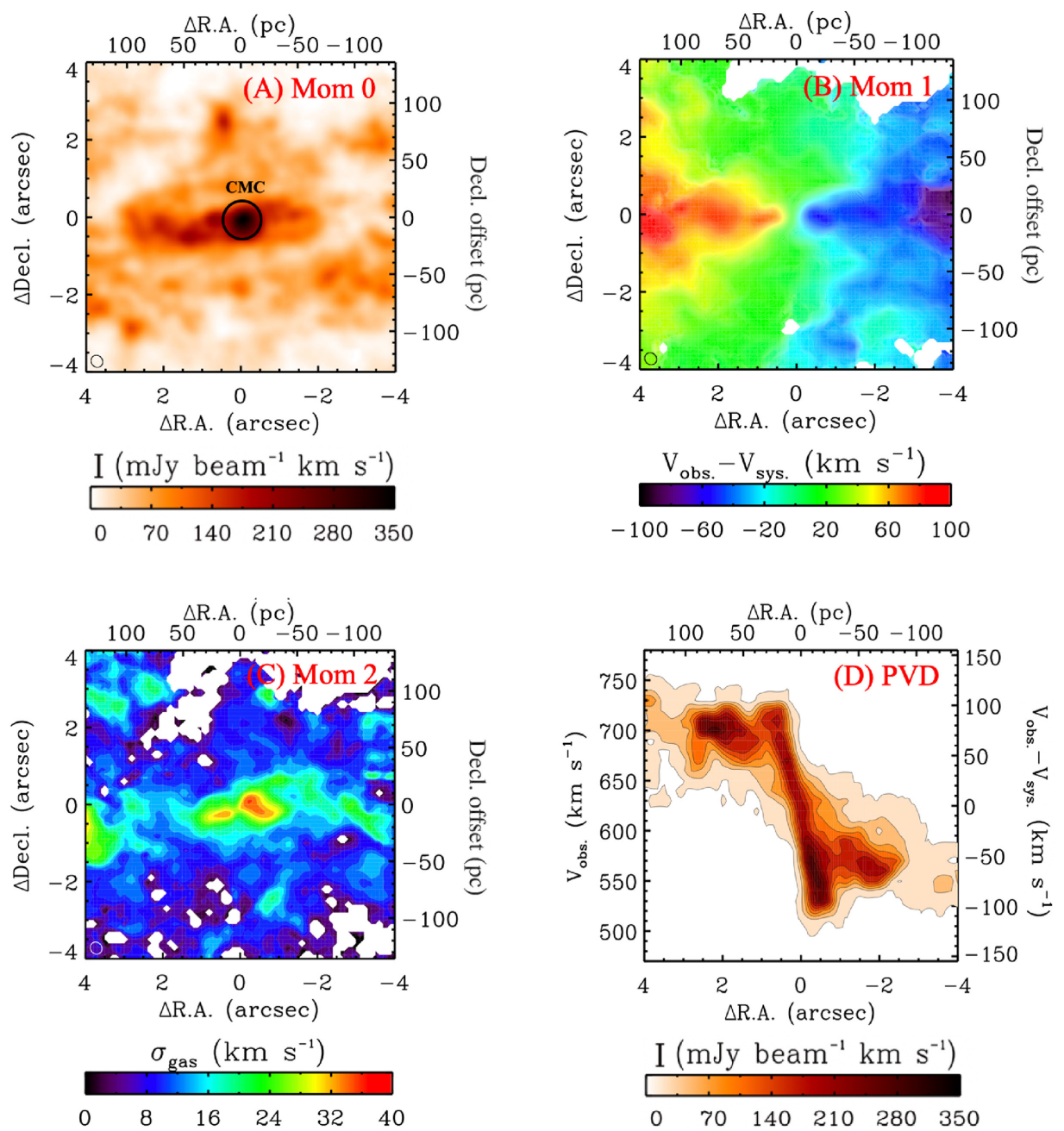

Figure 2. Zoomed-in moment maps and PVD of the ${ }^{12} \mathrm{CO}(2-1)$ emission of NGC 3593 with a field-of-view of $\approx 4 \times 4 \operatorname{arcsec}^{2}\left(\approx 140 \times 140 \mathrm{pc}^{2}\right)$, illustrating the morphology and kinematics of the CND in the vicinity of the central SMBH. As for Figs B1 and B3 in Appendix B, the panels include the integrated intensity map (Panel A; the CMC is clearly visible at the galaxy centre and is indicated by a black circle), intensity-weighted mean LOS velocity map (Panel B) and intensity-weighted LOS velocity dispersion map (panel C), as well as the kinematic major-axis PVD (Panel D). The synthesized beam (0!'33 $\times 0$ '”29 or $\left.11.6 \times 10.2 \mathrm{pc}^{2}\right)$ is shown as a tiny black or white ellipse in the bottom left-hand corner of Panels A-C.

discs from Coccato et al. (2013), convert the average into MGE form, and scale it by a (free) mass surface density at a radius of 4 arcsec $\left(\Sigma_{\star, 4^{\prime \prime}}\right)$. Here, we exclude the inner region $(r<4 \operatorname{arcsec})$ of the averaged mass surface-density profile to avoid doubly counting its mass later, when scaling the inner part (Section 4.3) to the outer part ( $4 \leq r \leqslant 30 \operatorname{arcsec}$ ) at this radius of $r=4 \operatorname{arcsec}$ (Section 4.4).

Second, because interstellar material (i.e. gas and dust) within the fitting region contributes significantly to the total mass and thus has a large impact on the fitting results, we must also include it in our mass model. Since the nucleus of NGC 3593 was classified as an H II star-forming region ionized by young massive stars (i.e. undergoing a burst of star formation; Hunter et al. 1989), we convert the ${ }^{12} \mathrm{CO}(2-$ 1) integrated intensity map to a molecular gas surface-density map by assuming a line ratio (in temperature units) ${ }^{12} \mathrm{CO}(2-1) /{ }^{12} \mathrm{CO}(1-$ $0)=0.8$ (Bigiel et al. 2008) and a CO-to- $\mathrm{H}_{2}$ conversion factor for starburst galaxies $X_{\mathrm{CO}}=(1.0 \pm 0.3) \times 10^{20} \mathrm{~cm}^{-2}\left(\mathrm{~K} \mathrm{~km} \mathrm{~s}^{-1}\right)^{-1}$ (Kuno et al. 2000, 2007; Bolatto, Wolfire \& Leroy 2013). This yields a total molecular gas mass $M_{\mathrm{H}_{2}}=(2.8 \pm 1.2) \times 10^{8} \mathrm{M}_{\odot}$, $\approx 40$ times smaller than the total stellar mass of the galaxy (see Sections 2 and 6.2). Hunter et al. (1989) reported a ratio of total atomic-to-molecular gas mass $M_{\mathrm{HI}} / M_{\mathrm{H}_{2}}=0.5$ (thus also consistent
Table 3. ISM MGE model.

\begin{tabular}{lccc}
\hline$j$ & $\log \left(\Sigma_{\mathrm{ISM}, j} / \mathrm{M}_{\odot} \mathrm{pc}^{2}\right)$ & $\sigma_{j}(\operatorname{arcsec})$ & $q_{j}$ \\
$(1)$ & $(2)$ & $(3)$ & $(4)$ \\
\hline 1 & 3.85 & 0.35 & 0.72 \\
2 & 3.13 & 2.42 & 0.64 \\
3 & 2.04 & 13.00 & 0.60
\end{tabular}

Note. Central ISM mass surface density $\left(\Sigma_{\mathrm{ISM}, j}\right)$, width $\left(\sigma_{j}\right)$ and axial ratio $\left(q_{j}\right)$ of each deconvolved Gaussian component $j$.

with the Pogge \& Eskridge 1993 H I measurement), that we adopt here (adding the atomic to the molecular hydrogen). For the dust, we adopt the total mass mentioned in Section $2\left(M_{\text {dust }}=10^{6} \mathrm{M}_{\odot}\right)$ and again add it to the molecular hydrogen. Lastly, we assume that the $\mathrm{H}_{2}, \mathrm{HI}$, and dust are all distributed according to the ${ }^{12} \mathrm{CO}(2-1)$ integrated intensity. Next, we again utilize the MGE formalism to decompose this total ISM (molecular hydrogen, atomic hydrogen and dust) map into individual Gaussian components, that are listed in Table 3 and are fixed (no free parameter).

Because the NGC 3593 molecular gas surface brightness cannot be described by a simple analytic function (with few free parameters), 


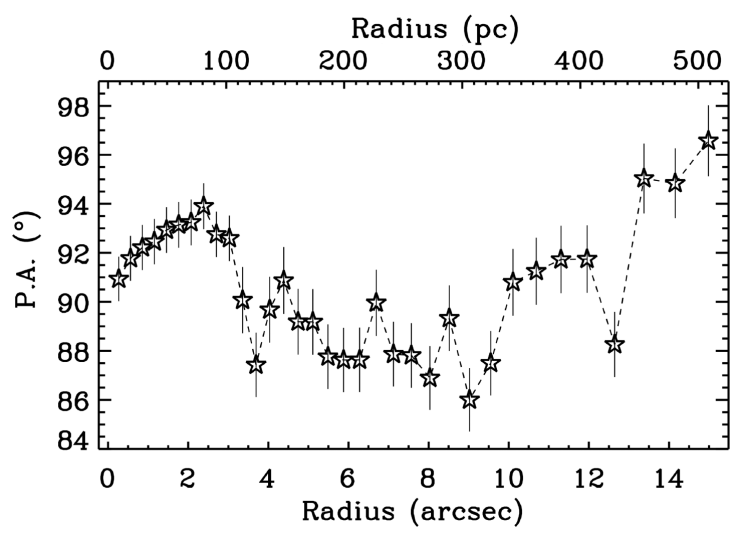

Figure 3. Radial position angle profiles of the ${ }^{12} \mathrm{CO}(1-0)$ emission, derived using the Kinemetry code of Krajnović et al. (2006).

and although this leads to a slight inconsistency between the ISM mass model above and the ${ }^{12} \mathrm{CO}(2-1)$ surface-brightness model, we use the SkySampler ${ }^{6}$ tool (Smith et al. 2019) for the fit. As the SkySampler clouds are created from the clean components of the cube, this is essentially equivalent to fitting only the kinematics of the molecular gas but not its distribution. The model thus matches the observed gas distribution with a single free parameter, the total flux $f$, used to rescale the entire cube (as the clouds are only assigned relative intensities by SkySampler). As the clean components do not include cleaning residuals, the total flux of the clean components is slightly lower than that of the cube, and $f$ also allows the model to recover this missing flux (although assuming that its distribution matches that of the clean components). The parameter $f$ should then simply be equal to the integrated flux of the (fitted region of the) cube, which serves as a useful sanity check on the model.

Lastly, we try to account for the small kinematic twist present in the ${ }^{12} \mathrm{CO}(1-0)$ intensity-weighted mean LOS velocity map (Panel B of Fig. 2 and Panel C of Fig. B1 in Appendix B), by first extracting the radial profile of the kinematic PA using the Kinemetry ${ }^{7}$ code of Krajnović et al. (2006), then using it as an additional model input along with the circular velocity curve. This kinematic PA profile varies only slightly $\left(86^{\circ}-96^{\circ}\right)$ across 15 arcsec, as shown in Fig. 3, but accounting for it in our dynamical model does help to reproduce the kinematic twist observed (see also Nguyen et al. 2020, 2021).

Overall, the model thus optimizes the fit to the observations with seven free parameters: stellar mass surface density at a radius of 4 $\operatorname{arcsec}$ (stellar mass normalization) $\Sigma_{\star, 4^{\prime \prime}}$, total flux of the fitted region (ISM mass normalization) $f$, inclination $i$, and galaxy centre in space $\left(x_{\mathrm{c}}, y_{\mathrm{c}}\right)$ and velocity $\left(v_{\text {off }}\right.$; all defined with respect to the previously determined centre; see Section 3.4), to which we add a spatially constant tracer (turbulent) velocity dispersion $\left(\sigma_{0}\right)$. The kinematic tracer is assumed to lie in a thin disc (by fixing the disc thickness $d_{\mathrm{t}}=$ 0; Davis et al. 2020). At this point, while modelling the outer part of the ${ }^{12} \mathrm{CO}(2-1)$ gas kinematics only, we ignore the gravitational potential contributed by the central SMBH (but see Section 4.3).

We run the KinMS fit in an area of 300 spaxels $\times 100$ spaxels $(30$ $\times 10$ arcsec) but exclude the inner 4 arcsec ( 40 spaxels $\times 40$ spaxels) so that the central masses (NSC, CMC, and SMBH) do not affect the outer mass surface-density profile. We will constrain the inner mass surface density independently in Section 4.3. We select a velocity

${ }^{6}$ https://github.com/Mark-D-Smith/KinMS-skySampler

${ }^{7}$ http://davor.krajnovic.org/idl/\#kinemetry range of 30 channels ( -150 to $150 \mathrm{~km} \mathrm{~s}^{-1}$ ) to cover the whole CND and adopt flat priors over reasonable parameter ranges, except for $\Sigma_{\star, 4^{\prime \prime}}$ for which we adopt a flat prior in logarithmic space (to ensure efficient sampling of the posterior). The chain performs $10^{5}$ calculations, the first 40 per cent of which are considered a 'burnin phase' and are excluded from the full MCMC. The remaining 60 per cent of the iterations are used to produce the final posterior probability distributions of the free parameters.

The best-fitting parameters and their uncertainties are identified directly from this Bayesian analysis, relying on the likelihood probability distribution functions (PDFs) generated via MCMC. We adopt the median of each posterior PDF as the best fit for that parameter, nearly identical to the minimum $\chi^{2}(<3$ per cent difference in all cases).

As discussed by van den Bosch \& van de Ven (2009), the statistical uncertainties can be severely underestimated when working with very large data sets, as systematic uncertainties starts to dominate over statistical ones. They thus proposed an approximate correction, based on the assumption that systematic uncertainties are similar to statistical ones. Accordingly, they suggested increasing the $\chi^{2}$ difference $\left(\Delta \chi^{2}\right)$ required to define a given confidence level by the standard deviation of the $\chi^{2}$ itself, namely $\sqrt{2(N-P)} \approx \sqrt{2 N}$ (where $N$ is the number of constraints and $P$ is the number of free model parameters, here $P=7$; see e.g. section 15.1 of Press 2007). When working with Bayesian methods rather than $\chi^{2}$ statistics, an equivalent effect can be achieved by dividing the model log likelihood by $\sqrt{2 N}$ or equivalently multiplying the measurement uncertainties (RMS) by $(2 N)^{1 / 4}$, as done by Mitzkus, Cappellari \& Walcher (2017). The dominance of systematic uncertainties over statistical ones is a generic issue with ALMA data cubes, due to the very large number of high signal-to-noise ratio constraints. This rescaling approach was therefore adopted in a number of recent papers using KinMS and ALMA data (e.g. Nagai et al. 2019; North et al. 2019; Smith et al. 2019, 2021b; Davis et al. 2020; Nguyen et al. 2020, 2021), to yield more realistic uncertainties.

The best-fitting model has a reduced $\chi^{2}\left(\chi_{\text {red }}^{2}\right)$ of $\approx 1.15$ for $N=$ $(300 \times 100-40 \times 40) \times 30=852000$ constraints (multiplying the uncertainties by $(2 N)^{1 / 4} \approx 36$ when calculating $\left.\chi_{\text {red }}^{2}\right)$. This relatively high $\chi_{\text {red }}^{2}$ (given the large number of constraints) is primarily due to the assumption of axisymmetry of the ISM mass distribution and mismatches between model and data in the high-velocity wings of the observed LOS velocity distributions at $\mid \Delta$ Position $\mid=4-8$ arcsec (see e.g. the observed PVD overlaid with the best-fitting model in the inset at the top right-hand panel of Fig. B4 in Appendix B). However, the fit provides an adequate description of the ${ }^{12} \mathrm{CO}(2-1)$ emission distribution and kinematics in the outer parts of the CND ( $r \geq 4 \mathrm{arcsec}$ ), and thus of the total mass distribution of the galaxy in that region. The seven best-fitting outer mass model parameters and their statistical uncertainties are listed in Table A1 in Appendix A, while their PDFs and two-dimensional (2D) marginalizations are shown in the corner plot of Fig. B4 in Appendix B. All parameters are well constrained and there is no strong covariance.

\subsection{Inner mass model}

Significant colour variation is seen in the nucleus of NGC $3593(r<$ $4 \operatorname{arcsec}$ or $r<140 \mathrm{pc}$ ) due to the two stellar populations aligned along the major axis (Coccato et al. 2013) and dust extinction (primarily to the north of the nucleus). We therefore construct a HST/WFPC2 $F 450 \mathrm{~W}-F 814 \mathrm{~W}$ colour map to create a mass-to-light ratio $(M / L)$ map based on the approximation of $F 450 \mathrm{~W}$ and $F 814 \mathrm{~W}$ to $g$ and $i$ 
band, respectively. The images are first astrometrically aligned and spatially convolved to match the PSFs, to mitigate potential spurious colour gradients near the galaxy centre (see Seth et al. 2010; Nguyen et al. 2017, 2018, 2019). The background level of each image is then estimated in small regions as far away from the galaxy centre as possible (radial range $13-15$ arcsec or $455-525 \mathrm{pc}$ ) and subtracted off. The inner $10 \times 10 \operatorname{arcsec}\left(350 \times 350 \mathrm{pc}^{2}\right)$ of the resulting colour map is shown in panel A of Fig. B5 in Appendix B. There is a clear colour dichotomy between the northern and southern halves (with respect to the galaxy centre).

As the stellar populations (e.g. ages and metallicity) in the nucleus of NGC 3593 are similar to those in the nucleus of NGC 5206 (see figs 7 and 12 of Kacharov et al. 2018), we follow the procedure of Nguyen et al. $(2017,2018)$ and use the Roediger \& Courteau (2015) colour $-M / L$ relation derived from stellar population synthesis models to estimate galaxy stellar masses (Nguyen et al. 2019). Such a correlation between colour and $M / L$ allows to calculate the $M / L$ (and then the stellar mass) based on colour information without knowing the detailed stellar populations and internal ISM extinction. Here, to convert the F450W-F814W (taken as $\approx g-i$ ) colour map to a $M / L_{F 814 \mathrm{~W}}$ map (Panel B of Fig. B5 in Appendix B), we adopt the relationship that assumes a Chabrier initial mass function (IMF) and a dust attenuation in molecular clouds and the ambient ISM described by Charlot \& Fall (2000). Correcting for Galactic foreground extinction and utilizing the photometric zero points listed in Table 2 and the HST/WFPC2 F814W Vega magnitude system, ${ }^{8}$ the multiplication of this $M / L_{F 814 \mathrm{~W}}$ map by the $F 814 \mathrm{~W}$-band luminosity surface-density map (Panel C of Fig. B5 in Appendix B) yields our desired stellar-mass surface-density map (Panel D of Fig. B5 in Appendix B). As clearly seen in the colour map, the central pixels at the putative NSC's location are redder than the surrounding galaxy, resulting in an NSC $M / L\left(M / L_{\mathrm{NSC}}\right)$ a factor 5-10 higher than that of the surrounding galaxy.

As the Charlot \& Fall (2000) dust and ISM attenuation prescription is fixed, such a high $M / L_{\mathrm{NSC}}$ is most easily understood as high obscuration at short wavelengths in the nucleus. However, it is possible that some of this attenuation is not accounted for accurately, leading to a biased $M / L_{\mathrm{NSC}}$. Indeed, we caution that the resulting $M / L$ map is dependent on the aforementioned assumptions and is thus subject to uncertainties. According to Roediger \& Courteau (2015), the error budget of the $M / L$ map (and thus the resulting stellar-mass surface density) is dominated by the stellar population modelling assumptions, with a colour-dependent bias of up to $0.3 \mathrm{dex}$ in the optical. Precise constraints on the $M / L$ and ISM attenuation $\left(\tau_{V}\right)$ require optical long-slit (Nguyen et al. 2017, 2019) or integral-field (Mitzkus et al. 2017; Thater et al. 2019) spectroscopic data, but no such datum is publicly available for NGC 3593.

In any case, we then again describe (i.e. parametrize) the resulting stellar-mass surface-density map using a MGE model. Here we use the procedure mge_fit_sectors_regularized (Cappellari 2002; see footnote 4) and constrain the allowable axial ratio (q) range to $0.39-0.95$, to avoid over-constraining the inclination of the ${ }^{12} \mathrm{CO}(2-1) \mathrm{CND}$ during modelling. Due to the significant dust extinction on the northern side of NGC 3593, the mass surfacedensity distribution remains highly asymmetric. We thus exclude all the pixels on the northern side during the MGE axisymmetric fit. A comparison of the mass surface density (black contours) and its MGE parametrization (red contours) is shown in Fig. B6 in Appendix B for the southern half of the galaxy.

\footnotetext{
${ }^{8}$ http://mips.as.arizona.edu/ cnaw/sun.html
}

Table 4. Combined stellar-mass F814W MGE model of NGC 3593.

\begin{tabular}{lccc}
\hline$j$ & $\log \left(\Sigma_{\star, j} / \mathrm{M}_{\odot} \mathrm{pc}^{2}\right)$ & $\sigma_{j}(\operatorname{arcsec})$ & $q_{j}$ \\
$(1)$ & $(2)$ & $(3)$ & $(4)$ \\
\hline \multicolumn{4}{c}{ Inner component } \\
1 & 5.15 & 0.06 & \\
2 & 4.89 & 0.13 & 0.95 \\
3 & 4.57 & 0.18 & 0.95 \\
& & & 0.95 \\
4 & Outer component & 0.38 & \\
5 & 4.22 & 1.25 & 0.39 \\
6 & 3.61 & 2.52 & 0.87 \\
7 & 3.54 & 3.96 & 0.39 \\
8 & 3.79 & 12.56 & 0.95 \\
& 3.72 & & 0.45 \\
\hline
\end{tabular}

Note. All quantities as in Table 3.

\subsection{Combined stellar-mass and total mass models}

Now that we have both inner $(r<4$ arcsec) and outer $(4 \leq r \lesssim$ $30 \mathrm{arcsec})$ stellar-mass surface-density models, we scale the inner model (i.e. panel D of Fig. B5 in Appendix B; see Section 4.3) to match the outer model (i.e. the two stellar discs averaged and scaled by the normalization factor $\Sigma_{\star, 4^{\prime \prime}}$; see Section 4.2) at the boundary ( $r=4 \operatorname{arcsec}$ or $140 \mathrm{pc}$ ), as the outer model was constrained more accurately by modelling the outer ${ }^{12} \mathrm{CO}(2-1)$ kinematics. This simultaneously allows to (1) recalibrate the sky backgrounds previously subtracted from the HST images, that were necessarily contaminated by galaxy light due to the small fields of view, and (2) avoid counting twice the inner stellar mass within 4 arcsec mentioned in Section 4.2. We note that the outer stellar-mass surface-density model therefore affects the inner mass surface-density model, that in turn has a strong influence on the inferred SMBH mass (see Section 5.2.1). Indeed, the scaling of the inner stellar-mass surfacedensity model to the outer stellar-mass surface-density model at the radius of 4 arcsec yields a combined stellar-mass surface-density model extending to at least $\approx 30 \mathrm{arcsec}$, that we use for all subsequent dynamical modelling.

The top panel of Fig. B7 in Appendix B shows a major-axis cut of this combined stellar-mass surface-density model in the form of two truncated MGE models (black and blue open squares), overlaid with its best-fitting MGE parametrization (red solid line). We note that this combined stellar-mass surface-density cut is not an observable, but was rather constructed from the outer (blue open squares; Section 4.2) and inner (black open squares; Section 4.3) stellar-mass surface-density maps. The fractional difference between the cut and its best-fitting MGE model is also shown in the bottom panel of Fig. B7 in Appendix B and is $\lesssim 10$ per cent at all radii. The individual components of this combined stellar-mass surface-density MGE model are listed in Table 4.

Given an inclination, any MGE Gaussian component can be deprojected analytically. Applying this to both our combined stellar (Table 4) and ISM (Table 3) MGE mass models yields a 3D total (stars + ISM) mass volume-density model of NGC 3593.

\section{BLACK HOLE MASS MEASUREMENT}

\subsection{Results}

We henceforth use our total MGE mass model (ISM and stars; Tables 3 and 4) to constrain the mass of the SMBH in the nucleus of NGC 3593, utilizing KinMS kinematic modelling analogous to that described in Section 4.2. However, the stellar mass surface density 
Table 5. Model parameters best fitting the inner part of the ${ }^{12} \mathrm{CO}(2-1)$ disc.

\begin{tabular}{lcccc}
\hline Parameter & Search range & Best fit & $\begin{array}{c}1 \sigma \text { uncertainty } \\
(1-84 \%)\end{array}$ & $\begin{array}{c}3 \sigma \text { uncertainty } \\
(0.14-99.86 \%)\end{array}$ \\
$(1)$ & $(2)$ & $(3)$ & $(4)$ & $(5)$ \\
\hline Black hole & & & & \\
$\log \left(M_{\mathrm{BH}} / \mathrm{M}_{\odot}\right)$ & $(1 \rightarrow 9)$ & 6.38 & $-0.08,+0.07$ & $-0.25,+0.25$ \\
$\Gamma$ & $(0.1 \rightarrow 2.0)$ & 0.89 & $-0.01,+0.02$ & $-0.03,+0.06$ \\
Gas CND & & & & \\
$f\left(\mathrm{Jy} \mathrm{km} \mathrm{s}^{-1}\right)$ & $\left(10^{2}-5 \times 10^{3}\right)$ & 1216.00 & $-0.17,+0.18$ & $-0.57,+0.59$ \\
$\sigma_{0}\left(\mathrm{~km} \mathrm{~s}^{-1}\right)$ & $(1 \rightarrow 50)$ & 15.01 & $-0.14,+0.15$ & $-0.45,+0.45$ \\
$i\left({ }^{\circ}\right)$ & $(70 \rightarrow 90)$ & 74.99 & $-0.14,+0.12$ & $-0.55,+0.50$ \\
Nuisance & & & & \\
$x_{\mathrm{c}}\left(^{\prime \prime}\right)$ & $(-1.0 \rightarrow 1.0)$ & 0.00 & $-0.04,+0.04$ & $-0.12,+0.12$ \\
$y_{\mathrm{c}}\left(^{\prime \prime}\right)$ & $(-1.0 \rightarrow 1.0)$ & 0.00 & $-0.03,+0.03$ & $-0.09,+0.09$ \\
$v_{\text {off }}\left(\mathrm{km} \mathrm{s}^{-1}\right)$ & $(-50 \rightarrow 50)$ & 32.98 & $-0.16,+0.14$ & $-0.50,+0.45$ \\
\hline
\end{tabular}

Notes. Same as Table A1 in Appendix A but for the inner part of the ${ }^{12} \mathrm{CO}(2-$ 1) $\mathrm{CND}$, and with the central SMBH mass $\left(M_{\mathrm{BH}}\right)$ and mass-scaling factor $(\Gamma)$ instead of the stellar-mass surface density at $4 \operatorname{arcsec}\left(\Sigma_{\star, 4^{\prime \prime}}\right)$. Here, we also list the uncertainties at the $1 \sigma$ confidence level.

at $4 \operatorname{arcsec}\left(\Sigma_{\star, 4^{\prime \prime}}\right)$ is no longer a free parameter (it is now fixed by the fitting of the outer kinematics; see Section 4.2), and we must introduce two new free parameters: the central SMBH mass $\left(M_{\mathrm{BH}}\right.$, with a flat prior in logarithmic space) and a stellar-mass scaling factor $\Gamma$, effectively the ratio of the dynamical and stellar population mass-tolight ratios $\left(\Gamma \equiv(M / L)_{\mathrm{dyn}} /(M / L)_{\mathrm{pop}}\right)$, thus scaling the stellar potential of the galaxy. We also leave the disc inclination $i$ to vary to explore any possible variation associated with the inclusion of the inner 4 arcsec CND. Thus, this final KinMS model used to constrain the central SMBH mass (and optimised to fit the molecular gas observations at all radii) has eight free parameters: $M_{\mathrm{BH}}, \Gamma, f, i, x_{\mathrm{c}}, y_{\mathrm{c}}, v_{\mathrm{off}}, \sigma_{0}$. We run the model in the same manner as in Section 4.2, with a total number of iterations of $10^{5}$ and the first 40 per cent of the iterations considered as the burn-in phase, yielding our final posterior PDFs.

We infer an SMBH mass that causes increasing rotation toward the centre as the radius decreases, although the NSC and CMC also contribute significant mass there. In fact, the significant contributions of these two compact components can be seen in the PVDs of the inset in Fig. B4 in Appendix B (model with the CMC but no NSC nor SMBH) and Panel A of Fig. B8 in Appendix B (model with the CMC and NSC but no SMBH), that show models without an SMBH overlaid on the data. The best-fitting model without an SMBH $\left(M_{\mathrm{BH}}=0 \mathrm{M}_{\odot}, \Gamma=0.92\right.$ and $i=75.45$; with $\left.\chi_{\text {red }}^{2} \approx 1.04\right)$ does not fit the data well in the centre, and it is clear that the observed molecular gas kinematics call for a central SMBH.

As listed in Table 5, the best-fitting KinMS model with an SMBH has $\left(M_{\mathrm{BH}}, \Gamma, i\right)=\left(2.40_{-1.05}^{+1.87} \times 10^{6} \mathrm{M}_{\odot}, 0.89_{-0.03}^{+0.06}, 74.99_{-0.55}^{+0.50}\right)$ with $\chi_{\text {red }}^{2} \approx 1.01$ (all uncertainties are quoted at the $3 \sigma$ statistical level), and the central molecular gas kinematics are now well reproduced. The resulting PVD is shown in panel B of Fig. B8 in Appendix $\mathrm{B}$, overlaid on the data. For comparison, we also show in Panel C of Fig. B8 in Appendix B a KinMS model with an overly massive $\operatorname{SMBH}\left(M_{\mathrm{BH}}=5.02 \times 10^{6} \mathrm{M}_{\odot}, \Gamma=0.87\right.$ and $\left.i=74.07\right)$ with $\chi_{\text {red }}^{2} \approx 1.06$, that again does not compare well to the data in the centre. We note that for the two models shown in Panels A and $\mathrm{C}$ of Fig. B8 in Appendix B, we varied $M_{\mathrm{BH}}, \Gamma$ and $i$ only while keeping the gas CND and other nuisance parameters fixed to those of the best-fitting model (see Table 5). The observed, best-fitting model and residual (data-model) ${ }^{12} \mathrm{CO}(2-1)$ mean LOS velocity maps are also shown in Panels D-F of Fig. B8 in Appendix B, respectively, to illustrate how well the model reproduces the data.
Additionally, the Panels G-I of Fig. B8 in Appendix B show the observed, best-fiting model and residual maps of the ${ }^{12} \mathrm{CO}(2-1) \mathrm{LOS}$ velocity dispersion, respectively, while the Panels J-L of Fig. B8 in Appendix B show the analogous integrated intensity maps. At least some of the turbulent/non-circular motions visible in the residual velocity map (Panel F of Fig. B8 in Appendix B) and unaccounted for by our (axisymmetric) model are associated with regions of high velocity dispersion (Panel G of Fig. B8 in Appendix B) and high surface brightness (Panel $\mathrm{J}$ of Fig. B8 in Appendix B), possibly indicating the presence of streaming gas (inflow and/or outflow), shocks, turbulence and/or filaments.

As only the very central region of $4 \times 4 \operatorname{arcsec}^{2}$ (or $\left.\approx 140 \times 140 \mathrm{pc}^{2}\right)$ of the ${ }^{12} \mathrm{CO}(2-1)$ kinematics matters to constrain the SMBH mass, we show a zoomed-in version of Fig. B8 in Appendix B (PVDs and mean LOS velocity maps only) in Fig. 4. The central rapidly rising velocities (as the radius decreases) of the molecular gas disc due to the central SMBH dominate within a radius $r \approx 0$ '.5 $(\approx 17.5 \mathrm{pc}$ ). However, the velocity residuals are significant (up to $\approx 20 \mathrm{~km} \mathrm{~s}^{-1}$ ) in an arc on the west side of the nucleus, which will be discussed further in Section 7.2.

For example, Fig. B9 in Appendix B shows the PDF and 2D marginalizations of each of the eight free parameters of our SMBH fit. The best-fitting model parameter is indicated by a vertical solid line in each PDF. The uncertainties resulting from the PDFs are indicated by vertical dashed lines at the $1 \sigma$ (16-84 per cent) confidence levels, while the contours in the $2 \mathrm{D}$ marginalizations show $0.5 \sigma$ (3169 per cent), $1 \sigma$ (16-84 per cent), $2 \sigma$ (2.3-97.7 per cent) and $3 \sigma$ (0.14-99.86 per cent) confidence levels. Most of the parameters are well constrained by the data, although as expected there is a significant covariance between $M_{\mathrm{BH}}$ and $\Gamma$, arising from the degeneracy between the potential of the SMBH and that of the stars and ISM when the observations do not adequately spatially resolve the SMBH's SOI.

We show in Fig. 5 the enclosed mass distributions (stars, ISM, and $\mathrm{BH}$ ) of our best-fitting model, and return to it later to gauge the robustness of this model.

Given the best-fitting $M_{\mathrm{BH}} \approx 2.4 \times 10^{6} \mathrm{M}_{\odot}$ and the central stellar velocity dispersion $\sigma_{\star} \approx 60 \mathrm{~km} \mathrm{~s}^{-1}(r \lesssim 5 \operatorname{arcsec}$ or $r \lesssim 175 \mathrm{pc}$; Bertola et al. 1996), the BH in NGC 3593 has a nominal SOI radius $R_{\mathrm{SOI}} \equiv G M_{\mathrm{BH}} / \sigma_{\star}^{2} \approx 3.0 \mathrm{pc}\left(\approx 00^{\prime} 09\right)$. The $R_{\mathrm{SOI}}$ is thus $\approx 3.5$ times smaller than what our ALMA observations (i.e. our synthesised beam of $\approx 0$ '.30) can spatially resolve.

Davis (2014), Barth et al. (2016a, 2016b), Boizelle et al. (2019, 2021), and Nguyen et al. (2020) demonstrated that the angular resolution $\theta_{\text {FWHM }}$ required to perform reliable $M_{\mathrm{BH}}$ measurements should satisfy $\theta_{\text {FWHM }} \lesssim 2 \times \theta_{\mathrm{R}_{\text {SOI }}}$, where $\theta_{\mathrm{R}_{\mathrm{SOI}}}$ is the angle subtended by $R_{\text {SOI. }}$. Measurements using data with poorer angular resolutions (i.e. larger synthesized beams) are more susceptible to systematic biases from stellar mass uncertainties. Our ALMA observations of NGC 3593 thus belong to the majority of $M_{\mathrm{BH}}$ measurements with ALMA and CARMA, that have $\theta_{\text {FWHM }} \gtrsim 2 \times \theta_{\text {RSOI }}$ (Davis et al. 2013, 2017; Onishi et al. 2015, 2017; Nagai et al. 2019; Smith et al. 2019, 2021b; Thater 2019; Nguyen et al. 2020, 2021). This suggests our $M_{\mathrm{BH}}$ estimate would benefit from observations at higher angular resolutions, to further reduce the uncertainties arising from our stellar-mass model (but see Section 5.2.1).

In addition to our limited synthesized beam (compared to the SMBH SOI), the (stellar) mass of the NSC is likely to be the greatest source of uncertainty on the SMBH mass, as it could be degenerate with $M_{\mathrm{BH}}$ (see items iii and iv in Section 5.2.1). However, it is worth noting here that the NSC is spatially resolved by the HST observations, which partially suppresses this stellar-mass un- 

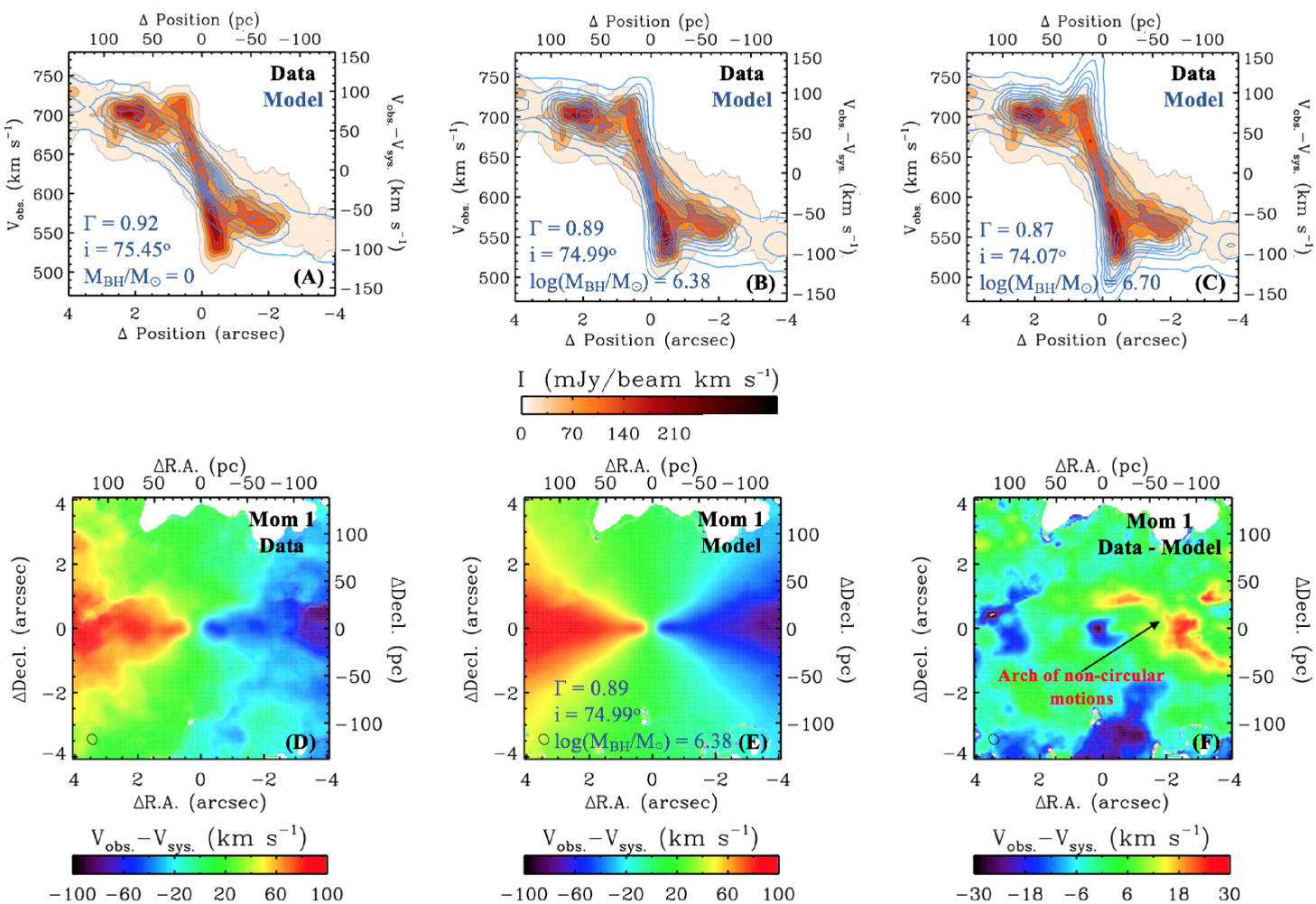

Figure 4. As the first two rows of Fig. B8 in Appendix B, but for a much smaller field-of-view of $4 \times 4 \operatorname{arcsec}^{2}\left(\approx 140 \times 140 \mathrm{pc}^{2}\right)$.

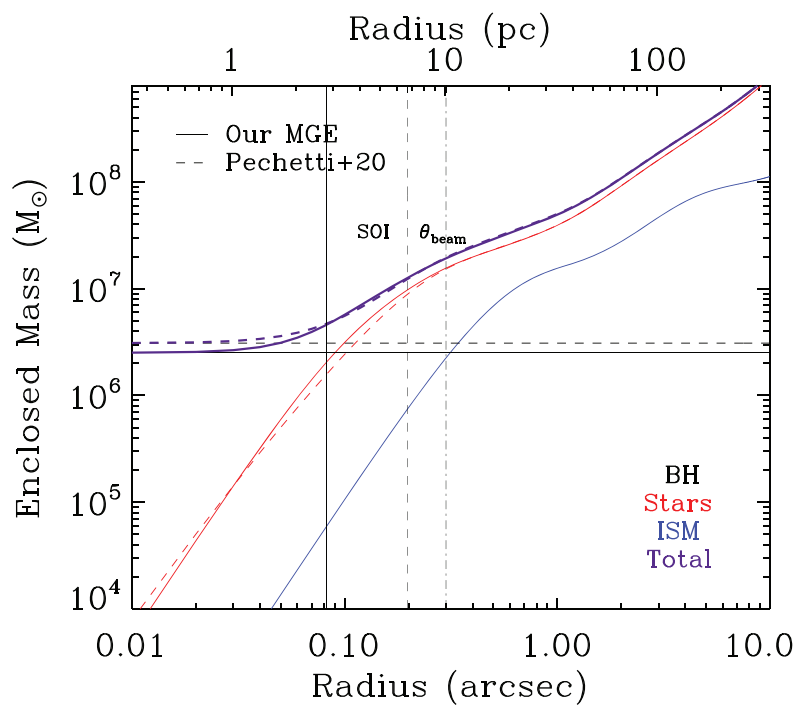

Figure 5. Cumulative mass distribution of the stars, ISM and $\mathrm{BH}$ of NGC 3593 for our best-fitting model (solid curves) and the NSC stellarmass model modified according to Pechetti et al. (2020) (dashed curves). The ISM (primarily the molecular gas) does not dominate the mass at any radius, but it does contribute significantly, especially at larger radii. The $R_{\text {SOI }}$ (as defined in the text) of our best-fitting model and the NSC-modified model are indicated by the vertical solid and dashed lines, respectively. The synthesized beam of $\approx 0^{\prime \prime} 3$ is indicated by the vertical dot-dashed line.

certainty/degeneracy and leads to a statistically significant rejection of the $M_{\mathrm{BH}}=0$ hypothesis. Indeed, the FWHM of the all three MGE components of the NSC mass model are greater than the PSF of the $H S T$ data $\left(\theta_{\mathrm{HST}} \approx 0^{\prime \prime}\right.$.08; $\mathrm{FWHM}_{j=1,2,3}=2.35 \sigma_{j=1,2,3}>\theta_{H S T}$; see
Table 6. Double Sérsic Fits.

\begin{tabular}{lcccc}
\hline $\begin{array}{l}\text { Comp. } \\
(1)\end{array}$ & $\begin{array}{c}r_{\mathrm{e}}(\mathrm{pc}) \\
(2)\end{array}$ & $\begin{array}{c}r_{\mathrm{e}}(\operatorname{arcsec}) \\
(3)\end{array}$ & $\begin{array}{c}n \\
(4)\end{array}$ & $\begin{array}{c}\text { Mass }\left(\times 10^{7} \mathrm{M}_{\odot}\right) \\
(5)\end{array}$ \\
\hline Stars \\
NSC & $5.0 \pm 1.0$ & $0.15 \pm 0.03$ & $1.0 \pm 0.1$ & $1.67 \pm 0.48$ \\
Disc & $567 \pm 30$ & $16.2 \pm 0.8$ & $1.4 \pm 0.2$ & $1,275 \pm 370$ \\
& \multicolumn{5}{c}{ ISM } \\
CMC & $11.2 \pm 2.8$ & $0.32 \pm 0.08$ & $1.1 \pm 0.1$ & $0.54 \pm 0.12$ \\
Disc & $444.5 \pm 3.5$ & $12.7 \pm 0.1$ & $1.0 \pm 0.1$ & $36.5 \pm 8.7$
\end{tabular}

Note. Columns 1-5 list each component's name, effective (half-light) radius in parsec and arcsecond, Sérsic index, and total mass, respectively.

Table 4$)$ and the NSC is almost spatially resolved $\left(r_{\mathrm{NSC}} \approx 2 \theta_{\mathrm{HST}}\right.$; see Table 6).

\subsection{Uncertainties}

We test here the robustness of our dynamical model (and the inferred best-fitting parameters) under the influence of sources of errors other than the uncertainties in the ALMA kinematics, $i$ and $\Gamma$ (discussed in Section 5.1). In these fits, except for $M_{\mathrm{BH}}, i$, and $\Gamma$, we fix all (CND and other nuisance) parameters to their best-fitting values listed in Table 5.

\subsubsection{Stellar mass models}

Our inner mass model constructed from HST images under the assumption of the colour $-M / L$ relation of Roediger \& Courteau (2015) (that assumes the Chabrier IMF and the Charlot \& Fall 2000 dust attenuation correction) has a large impact on our model results. We 

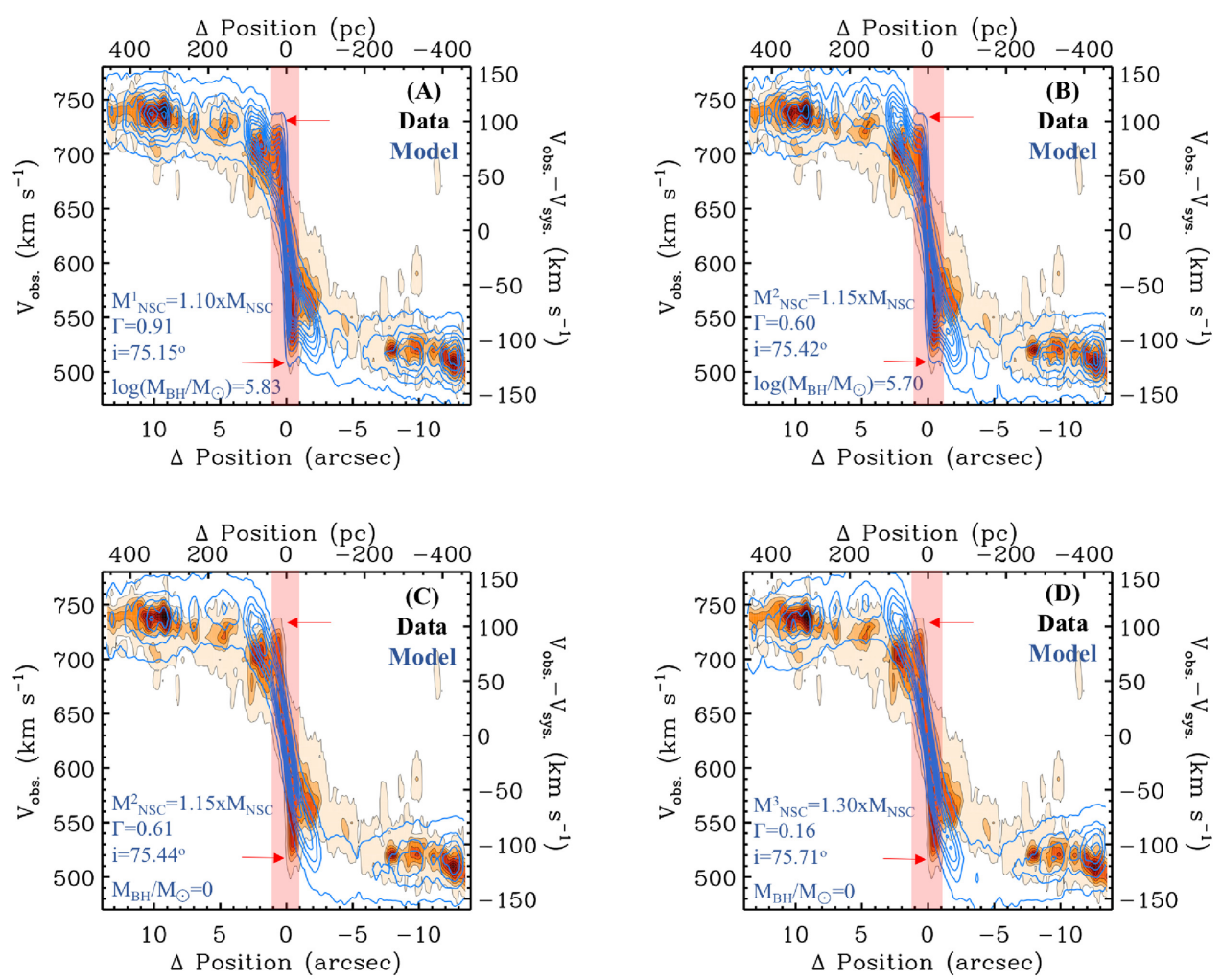

Figure 6. PVD of the ${ }^{12} \mathrm{CO}(2-1)$ emission of NGC 3593 extracted along the kinematic major-axis (orange scale and grey contours), overlaid with the modelled PVDs (blue contours) of the best-fitting models with different assumptions of NSC mass. The parameters of the best-fitting models are shown in the legends, while the red arrows indicate the rapidly rising rotation velocities (as the radius decreases) in the centre, putatively caused by a central SMBH. Although $M_{\mathrm{NSC}}$ and $\Gamma$ dictate the shapes of the PVDs at large radii, the central rising velocities require an SMBH.

therefore further examine the associated uncertainties, by considering other independent models constructed with different assumptions.

(i) Mass model without mask: To create our inner stellar-mass model, we masked out most of the northern side of the nucleus, that is strongly extincted by dust. We therefore create here another stellar-mass MGE model without masking.

(ii) Mass model using F450W filter: We also test a mass model created from the HST F450W (rather than F814W) image. As in Section 4.3, we use the Roediger \& Courteau (2015) colour-M/L relation to derive a $M / L_{F 450 \mathrm{~W}}$ map and in turn a $F 450 \mathrm{~W}$-based stellarmass map, and then parametrize the resulting stellar-mass map using MGE. We then use this F450W-based MGE stellar-mass model (rather than that in Table 4) in conjunction with KinMS for the kinematic modelling.

(iii) Degeneracy between our NSC mass model and $M_{\mathrm{BH}}$ : In Section 6.1, we will identify the summation of the three innermost MGE components of Table 4 as our NSC. That NSC is then 10 per cent smaller in size $\left(\approx R_{\mathrm{SOI}}\right)$ but 10 times smaller in mass than the NSC of Pechetti et al. (2020). As seen in Panels A of Figs B8 in Appendix B and 4, our KinMS model with an NSC $\left(M_{\mathrm{NSC}}=\right.$ $1.67 \times 10^{7} \mathrm{M}_{\odot}$ ) but no SMBH does not fit the data well in the centre (which requires a $\sim 10^{6} \mathrm{M}_{\odot} \mathrm{SMBH}$; see Panels B of Figs B8 in Appendix B and 4). This suggests that the masses of the NSC and SMBH of NGC 3593 may not be as degenerate as originally feared, at least on the spatial scale of $R_{\mathrm{SOI}}\left(R_{\mathrm{SOI}} \approx 3.0 \mathrm{pc}\right.$, see Section 5.1 ; $r_{\mathrm{NSC}, \mathrm{e}} \approx 5.0 \mathrm{pc}$, see Section 6.1 and Table 6 ).

We nevertheless test this with models with an SMBH and slightly increased NSC masses (or equivalently stellar-mass surface densities, i.e. increasing $\Sigma_{\star, j=1,2,3}$ but keeping $\sigma_{j=1,2,3}$ and $q_{j=1,2,3}$ fixed in Table 4), without increasing the more extended stellar-mass distribution (i.e. keeping the $j=4-8$ components fixed in Table 4). Specifically, we increase the NSC mass by factors of 1.10 (10 per cent, $M_{\mathrm{NSC}}^{1} \approx$ $\left.1.9 \times 10^{7} \mathrm{M}_{\odot}\right), 1.15\left(15\right.$ per cent, $\left.M_{\mathrm{NSC}}^{2} \approx 2.0 \times 10^{7} \mathrm{M}_{\odot}\right)$ and 1.30 (30 per cent, $M_{\mathrm{NSC}}^{3} \approx 2.2 \times 10^{7} \mathrm{M}_{\odot}$ ) and check for variations of the best-fitting $M_{\mathrm{BH}}$.

While the first new NSC mass $\left(M_{\mathrm{NSC}}^{1}\right)$ is chosen to yield a slightly smaller $M_{\mathrm{BH}}$, the second $\left(M_{\mathrm{NSC}}^{2}\right)$ is chosen to explore the possibility of an SMBH mass close to zero. The best-fitting results are listed in Table A2 in Appendix A and shown in panels A and B of Fig. 6 , indicating as expected that in both cases a good fit to the ${ }^{12} \mathrm{CO}(2-1)$ kinematics in the central $2^{\prime \prime} \times 2^{\prime \prime}$ region (see the red arrows in Fig. 6) requires an $\mathrm{SMBH}$ mass smaller than that of our default best-fitting model (see Section 5.1 and Table 5). However, in both cases, there are also significant mismatches between the data and the best-fitting model outside the centre $(1 \lesssim r \lesssim 7 \mathrm{arcsec})$, due to the increasing effect of the NSC's gravitational potential, and indeed the $M_{\mathrm{BH}} \mathrm{PDF}$ robustly excludes $M_{\mathrm{BH}}=0$ in both cases.

To further test the presence of an SMBH in our models, the third new NSC mass $\left(M_{\mathrm{NSC}}^{3}\right)$ is chosen to entirely remove the need for an SMBH. To achieve this, we choose a mass $\left(M_{\mathrm{NSC}}^{3} \approx 2.2 \times 10^{7} \mathrm{M}_{\odot}\right)$ equal to the highest NSC mass allowed by our default best-fitting model (see Table 6), nearly 10 times the default best-fitting SMBH mass. We show the best-fitting $M_{\mathrm{NSC}}^{3}$ model in panel D of Fig. 6, imposing $M_{\mathrm{BH}}=0$, and revealing that the rapidly rising rotation velocities of the ${ }^{12} \mathrm{CO}(2-1)$ gas in the centre (as the radius decreases) cannot be fit without an SMBH. Imposing $M_{\mathrm{BH}}=0$ for $M_{\mathrm{NSC}}^{2}$ as well, panel $\mathrm{C}$ of Fig. 6 shows that, as expected, the problem becomes 
more acute for smaller $M_{\mathrm{NSC}}$. In fact, focussing exclusively on the central $2 \times 2 \operatorname{arcsec}^{2}$ region (and ignoring the increasing mismatch at large radii), dynamical models with $M_{\mathrm{NSC}}=M_{\mathrm{NSC}}^{3}$ still require an SMBH with $M_{\mathrm{BH}}>3 \times 10^{5} \mathrm{M}_{\odot}$ to match the rapidly rising rotational velocities in the centre (see Table A2 in Appendix A).

Given the accuracy of our nuclear stellar-mass model based on the Roediger \& Courteau (2015) colour- $M / L$ relation (see Section 4.3 and Table 4) and the Sérsic fit to the NSC discussed in Section 6.1, these tests put a firm lower limit on the SMBH mass in the nucleus of NGC $3593\left(M_{\mathrm{BH}}>3 \times 10^{5} \mathrm{M}_{\odot}\right.$, including all possible uncertainties), and thus make our claim of a detection of an SMBH at the heart of NGC 3593 strong, this despite a synthesized beam size that is 3.5 times larger than $R_{\text {SOI }}$ (see Section 5.1).

(iv) Mass model of the NSC: In item (iii) above, we tested the impact of the NSC on our $M_{\mathrm{BH}}$ estimate by increasing its surface density and thus mass only (i.e. we varied $\Sigma_{\star, j}$ but kept $\sigma_{j}$ and $q_{j}$ fixed). Here, we further test this impact by considering variations of the NSC shape (i.e. $\sigma_{j}$ and $q_{j}$ ). For this, we replace the first three Gaussians of our (inner) stellar-mass MGE model (our NSC defined in item (iii) above; see Table 4) by the MGE components obtained from the product of the NSC Sérsic light profile and mass-to-light ratio of Pechetti et al. (2020) (listed in Table 1; see Section 6.1 for the reasons behind an order of magnitude difference between the mass of this assumed $M_{\mathrm{NSC}}$ and our own) and run our KinMS model again. The enclosed mass distributions (stars, ISM and BH) of this modified model is shown in Fig. 5, compared to that of our best-fitting model. The two profiles are nearly indistinguishable (including statistically consistent $M_{\mathrm{BH}}$ ), clearly demonstrating that our $M_{\mathrm{BH}}$ and $M / L_{F 814 \mathrm{~W}}$ (i.e. $\Gamma$ ) estimates are robust against systematic but realistic changes to our NSC mass model (see also Section 6.1 and Table A2 in Appendix A).

The best-fitting parameters of the six modified models above are listed in Table A2 in Appendix A, along with those of our best-fitting model (Section 5.1 and Table 4), demonstrating that our results are robust against reasonable changes of the stellar-mass model.

\subsubsection{Constant $\mathrm{M} / \mathrm{L}$ and variable dust extinction}

We note that our approach so far has essentially assigned all colour variations to stellar population (and thus $M / L$ ) variations (although the Roediger \& Courteau 2015 colour- $M / L$ relation does have a prescription for dust attenuation in the ISM). However, there is significant dust extinction near the major axis in the nucleus $(|\Delta \mathrm{Dec}|$. $\lesssim 1$ arcsec; see panel A of Fig. B5 in Appendix B), leading to $M / L$ variations of a factor of a few in that region and up to a factor of $\approx 10$ in the NSC. It is unclear if these variations are indeed real, or if they could instead be due to dust that has been misaccounted for in the Roediger \& Courteau (2015) model. This could lead to a significant overestimate of the stellar-mass surface densities in the nucleus, and in turn an underestimate of the inferred SMBH mass.

Given that the galaxy likely contains two stellar populations in co-spatial counterrotating discs (see Section 2), it may be that the stellar populations (and intrinsic colours and $M / L$ ) are rather uniform across the nucleus. Here, we therefore assume that the stellar populations are indeed uniform and adopt a single intrinsic colour (and thus $M / L$ according to the Roediger \& Courteau 2015 colour- $M / L$ relation using the Chabrier IMF and Charlot \& Fall 2000 attenuation prescription) across the whole field-of-view (inner part), in effect assigning all colour variations to dust extinction.

The $F 450 \mathrm{~W}-F 814 \mathrm{~W}$ and $M / L_{F 814 \mathrm{~W}}$ maps (Panels $\mathrm{A}$ and $\mathrm{B}$ of Fig. B5 in Appendix B) suggest that the southern half of the nucleus is largely dust free. First, we therefore adopt the typical colour and
$M / L$ of that region $\left(F 450 \mathrm{~W}-F 814 \mathrm{~W} \approx 1.7 \mathrm{mag}\right.$ and $M / L_{F 814 \mathrm{~W}} \approx$ $\left.1.7 \mathrm{M}_{\odot} / \mathrm{L}_{\odot}, F 814 \mathrm{~W}\right)$ as our unique colour and $M / L$ for the entire FOV (inner part).

Next, we assume $F 450 \mathrm{~W} \approx B$ and $F 814 \mathrm{~W} \approx I$, adopt an intrinsic colour $F 450 \mathrm{~W}-F 814 \mathrm{~W} \approx(B-I)_{0} \approx 1.7 \mathrm{mag}$, and use another form of the Milky Way extinction law, ${ }^{9}$ i.e. $A_{I}=0.572 \times E(B-I)=$ $0.572 \times\left[(B-I)-(B-I)_{0}\right]$, to correct our $F 814 \mathrm{~W}$ map for this dust extinction pixel-by-pixel over the entire $F 450 \mathrm{~W}-F 814 \mathrm{~W}$ colour map (Panel A of Fig. B5 in Appendix B). For example, in the central region co-spatial with the NSC and CMC, $(B-I) \approx 3.6$ mag, yielding an $I$-band dust extinction $A_{I} \approx 1.1 \mathrm{mag}$. This process yields an $I$-band image corrected pixel-by-pixel for dust extinction, that we multiply by our unique $M / L_{I}$ pixel-by-pixel to get the stellar-mass surfacedensity map (corrected pixel-by-pixel for dust extinction), that we finally parametrize with MGE as usual.

Using this arguably extinction-free stellar-mass model, we re-run our KinMS kinematic model and obtain $M_{\mathrm{BH}}=1.54_{-0.57}^{+0.49} \times 10^{6} \mathrm{M}_{\odot}$, $\Gamma=0.80_{-0.15}^{+0.16}$ and $i=73.86_{-0.57}^{+0.78}$ (also listed in Table A2 in Appendix A). This suggests that colour variations purely due to dust extinction (i.e. a fixed stellar population and thus $M / L$ ) lead to a central SMBH mass $\approx 36$ per cent smaller than that of our default best-fitting model assigning colour variations primarily to stellar population (and thus $M / L$ ) variations. The mass scaling factor $\Gamma$ (or equivalently the stellar $M / L)$ is also correspondingly smaller by $\approx$ 10 per cent. Given our adopted colour $(F 450 \mathrm{~W}-F 814 \mathrm{~W} \approx 1.7 \mathrm{mag})$ and $M / L\left(M / L_{F 814 \mathrm{~W}} \approx 1.7 \mathrm{M}_{\odot} / \mathrm{L}_{\odot, F 814 \mathrm{~W}}\right)$ for the entire inner part of the FOV $(r<4 \operatorname{arcsec}$ or $r<140 \mathrm{pc}$ ), this lighter SMBH is expected. Indeed, the dust-extinction correction in the nucleus is then larger than that of our default model, leading to higher corrected (i.e. intrinsic) surface brightnesses and thus masses (including for the NSC), and thus to a smaller $M_{\mathrm{BH}}$.

\subsubsection{ISM disc}

Given our inferred SMBH mass and associated $R_{\mathrm{SOI}}(\approx 3.5$ times smaller than the synthesised beam), the ISM mass contained within the central beam $(\approx 0.3$ or $\approx 10.5 \mathrm{pc})$ is dynamically significant, $\approx 2 \times 10^{6} \mathrm{M}_{\odot}$ (see Fig. 5 ) or $\approx 83$ per cent of $M_{\mathrm{BH}}$ and slightly greater than the $3 \sigma M_{\mathrm{BH}}$ uncertainty (see Table A2 in Appendix A). We thus also test the impact of the ISM on the inferred $M_{\mathrm{BH}}$, removing the ISM whose mass model is listed in Table 3 (see Section 4.2) and turning on the gasGrav function in the KinMS fit, that assumes the ISM mass is distributed according to the input ${ }^{12} \mathrm{CO}(2-1)$ surfacebrightness profile (thus also removing the inconsistency between the ISM mass model and ${ }^{12} \mathrm{CO}(2-1)$ surface brightness model noted in Section 4.2). This test yields $M_{\mathrm{BH}}=2.1_{-0.77}^{+0.54} \times 10^{6} \mathrm{M}_{\odot}, \Gamma=$ $0.90_{-0.07}^{+0.07}$ and $i=75.05_{-0.54}^{+0.52}$ (also listed in Table A2 in Appendix A), almost identical and fully consistent with the results from our default best-fitting model, analogous to the behaviour reported by Nguyen et al. (2020). Our best-fitting $M_{\mathrm{BH}}$ is thus not sensitive to the ISM distribution, as long as the total ISM mass is accurately estimated.

\subsubsection{CO-to- $\mathrm{H}_{2}$ conversion factor}

NGC 3593 is classified as a starburst galaxy (Hunter et al. 1989), but has a CND SFR of $\$ 3 \mathrm{M}_{\odot}$ year $^{-1}$ (Ho et al. 1997), much lower than the typical SFR of starbursts $\left(10-100 \mathrm{M}_{\odot}\right.$ year $\left.^{-1}\right)$. This suggests that our adoption of the starbusrt CO-to- $\mathrm{H}_{2}$ conversion factor $\left(X_{\mathrm{CO}}=(1.0 \pm 0.3) \times 10^{20} \mathrm{~cm}^{-2}\left(\mathrm{~K} \mathrm{~km} \mathrm{~s}^{-1}\right)^{-1}\right.$; e.g. Kuno

${ }^{9} \mathrm{http}: / /$ www.astro.sunysb.edu/metchev/PHY517_AST443/extinction_lab.pdf 
et al. 2000, 2007; Bolatto et al. 2013) in Section 4.2 may be inappropriate and may underestimate the total molecular gas mass by a factor of $\approx 2$. We therefore test this hypothesis by adopting instead the conversion factor of the Milky Way $\left(X_{\mathrm{CO}}=(2.0 \pm 0.3)\right.$ $\times 10^{20} \mathrm{~cm}^{-2}\left(\mathrm{~K} \mathrm{~km} \mathrm{~s}^{-1}\right)^{-1}$; e.g. Bolatto et al. 2013), that is arguably better suited to targets hosting molecular gas reservoirs with SFR $\lesssim 3 \mathrm{M}_{\odot}$ year $^{-1}$. The best-fitting KinMS model then yields $M_{\mathrm{BH}}=1.51_{-0.54}^{+0.83} \times 10^{6} \mathrm{M}_{\odot}, \Gamma=0.82_{-0.12}^{+0.12}$ and $i=75^{\circ} .35_{-0.48}^{+0.50}$ (also listed in Table A2 in Appendix A), suggesting the choice of the conversion factor, and hence the derived molecular gas mass, has a significant effect on the SMBH mass determination (possibly slightly beyond the statistical uncertainties of our default model).

To test whether the ISM could altogether negate the need for an $\mathrm{SMBH}$, we also tested more extreme values of the conversion factor $X_{\mathrm{CO}}$, artificially increasing the total gas mass (via $X_{\mathrm{CO}}$ and thus $M_{\mathrm{H}_{2}}$ ) and adjusting $\Gamma$ (otherwise fixing the stellar mass model and $M_{\mathrm{H}_{\mathrm{I}}}$ ) to fit the CND kinematics. For the best-fitting model to allow $M_{\mathrm{BH}}=0$, a total gas mass $M_{\mathrm{H}_{2}}+M_{\mathrm{HI}} \gtrsim 3 \times 10^{9} \mathrm{M}_{\odot}$ is required, more than 10 times the galaxy's nominal gas mass. Such high conversion factors ( $>10$ times the norm) are out of the range typically expected for the metallicity and luminosity of NGC 3593 (Bolatto et al. 2013), thus confirming yet again the need for a central SMBH.

\subsubsection{Asymmetric drift correction}

Barth et al. (2016a) and Boizelle et al. (2019) have suggested that pressure support may significantly bias SMBH mass estimates obtained from modelling the kinematics of molecular gas, due to potentially high turbulent velocity dispersions. In NGC 3593, except for a few regions with ${ }^{12} \mathrm{CO}(2-1)$ LOS velocity dispersions $\sigma \approx 25-$ $33 \mathrm{~km} \mathrm{~s}^{-1}$ (coincident with clumps of high emission; see panel D of Fig. B1 in Appendix B and panel C of Fig. 2), the molecular gas velocity dispersion is generally low $\left(\sigma \approx 10-15 \mathrm{~km} \mathrm{~s}^{-1}\right)$. Compared to the observed rotation velocities $V_{\text {rot }}, \sigma / V_{\text {rot }}<0.1$ in the entire $\mathrm{CND}$, suggesting that the rotation velocities are similar to the circular velocities (i.e. $V_{\text {rot }} \approx V_{\text {circ }}$ ). Any asymmetric drift correction is thus likely to have a minimal impact on our results.

To more rigorously test this, given such small $\sigma / V_{\text {rot }}$, we assume that the random motions in the CND are approximately equal in the radial $\left(\sigma_{\mathrm{r}}\right)$ and vertical $\left(\sigma_{\mathrm{z}}\right)$ directions (i.e. $\left.\sigma_{\mathrm{r}}=\sigma_{\mathrm{z}}\right)$ and that both are equal to the observed velocity dispersion $\sigma\left(\left\langle V_{\mathrm{r}} V_{\mathrm{z}}\right\rangle=0\right)$. We can then calculate the ratio of the velocity dispersions in the radial and azimuthal directions $\sigma_{\mathrm{r}} / \sigma_{\phi}=\sigma / \sigma_{\phi}$ using the epicycle approximation (equations 4-33 of Binney \& Tremaine 1987), and the asymmetric drift correction can then be estimated as

$$
V_{\text {circ }}^{2}-V_{\text {rot }}^{2}=\sigma^{2}\left[-r \frac{\mathrm{d} \ln \Sigma_{\mathrm{H}_{2}}}{\mathrm{~d} r}-r \frac{\mathrm{d} \ln \sigma^{2}}{\mathrm{~d} r}-\left(1-\frac{\sigma^{2}}{\sigma_{\phi}^{2}}\right)\right] .
$$

This yields corrections $<20$ per cent, so we conclude that any asymmetric drift correction (and associated uncertainties) will be small compared to other potential sources of error in this low-mass object. In turn, this suggests that the thin disc assumption is good enough to describe the nuclear molecular gas of NGC 3593, consistent with our earlier assumption of thin disc of the SkySampler (see Section 4.2).

\section{CENTRAL CORES}

\subsection{Nuclear star cluster and massive core of cold molecular gas}

We hereby characterize the morphological properties of the CMC and NSC, estimating their masses and sizes and comparing them to

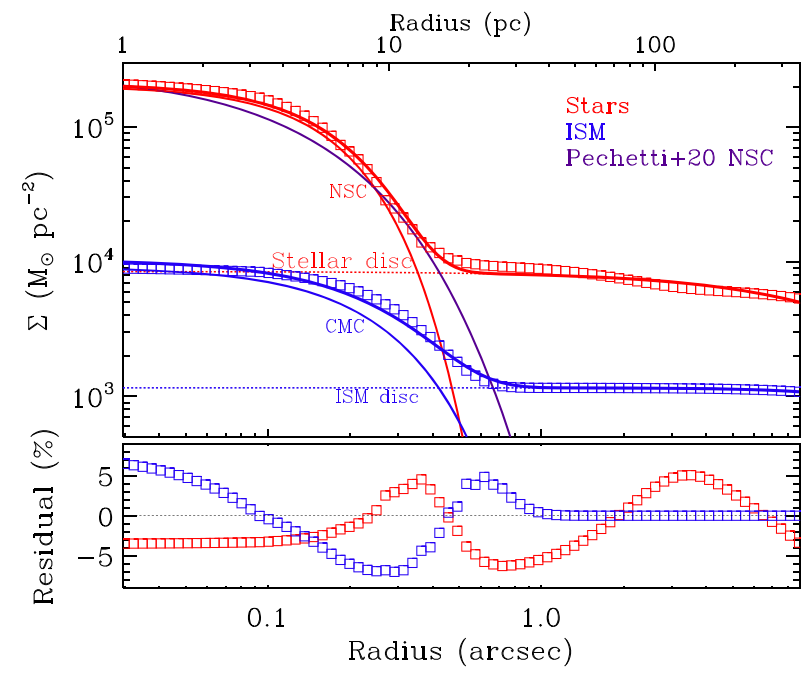

Figure 7. Top panel: Radial mass surface-density profile (open squares) of the stars (red) and ISM (blue) of NGC 3593, overlaid with the best-fitting narrow Sérsic component (thin solid line; respectively NSC and CMC), broad Sérsic component (dotted line; respectively stellar disc and ISM disc) and total profile (thick solid line) in matching colour (see Table 6). The purple thin line is the NSC of Pechetti et al. (2020). Bottom panel: fractional residuals [(data-model)/data].

estimates from our stellar and ISM (MGE) mass models. We use the Image Reduction and Analysis Facility (IRAF) ellipse task (Jedrzejewski 1987) to extract radial mass surfacedensity profiles of the stars (Panel D of Fig. B5 in Appendix B, i.e. F814W emission corrected for stellar populations and dust; see Section 4.3) and ISM (Panel B of Fig. B1 in Appendix B, i.e. ${ }^{12} \mathrm{CO}(2-1)$ emission corrected for $X_{\mathrm{CO}}, \mathrm{HI}$, and dust; Section 4.2) in concentric annuli with varying position angles and ellipticities, although keeping both fixed does not change our results. For convenience, we then fit both the stellar and the ISM radial mass surface-density profile with a double-Sérsic function. The fits are carried out using a non-linear least-squares algorithm (IDL MPFIT function; Markwardt 2009), and the results are shown in the top panel of Fig. 7. Before comparing the model and data, at each iteration the double-Sérsic function of the stars is first convolved by the F814W PSF and the double-Sérsic function of the ISM is first convolved by the synthesized beam of our ALMA observations, thus yielding spatially deconvolved (i.e. intrinsic) model (double-Sérsic function) parameters. We associate the narrow and the broad component with respectively the NSC and the outer stellar disc for the stellar-mass profile, and with the CMC and the ISM disc for the ISM-mass profile. Our best-fitting double-Sérsic models are good representations of the data, with fractional residuals smaller than 8 per cent [(data-model)/data; see the bottom panel of Fig. 7]. The best-fitting double-Sérsic parameters and associated total mass estimates are listed in Table 6.

Our newly derived NSC total stellar mass $\left(M_{\mathrm{NSC}}\right)$ is an order of magnitude smaller than that reported by Pechetti et al. (2020) $\left(M \approx 1.58 \times 10^{8} \mathrm{M}_{\odot}\right)$. This large discrepancy may be caused by several factors. First, Pechetti et al.'s (2020) spatially deconvolved photometric fit using the $F 814 \mathrm{~W}$ image likely suffers from heavy and uncorrected dust extinction in the nucleus. Indeed, they did not correct for dust but applied a mask and interpolated the northern half of the light distribution based on the southern half. Combined with their assumption of a constant and large $M / L\left(M / L \approx 5.5 \mathrm{M}_{\odot} / L_{\odot}\right)$, this may lead to a more massive stellar component. Second, Pechetti et al.'s (2020) NSC has a larger size ( $\left.r_{\mathrm{NSC}, \mathrm{e}}=5.50 \pm 0.23 \mathrm{pc}\right)$, and 
thus has more mass at large radii $(r>8 \mathrm{pc})$, than our newly derived $\operatorname{NSC}\left(r_{\mathrm{NSC}, \mathrm{e}}=5.0 \pm 1.0 \mathrm{pc}\right.$; see Fig. 7$)$. Third, Pechetti et al.'s (2020) adopted distance to NGC 3593 is $\approx 11$ Mpc (Karachentsev et al. 2004), > 50 per cent greater than our adopted distance, and hence they derive a significantly larger NSC mass (see footnote 2). Here, our stellar-mass map (and associated radial stellar-mass surface-density profile) accounts for all these effects and is thus arguably a more accurate representation of the true stellar-mass distribution.

For comparison, the total stellar mass and effective radius estimated from the three innermost Gaussian components in Table 4 are $r_{\mathrm{NSC}, \mathrm{e}}=0^{\prime \prime} 15 \pm 00^{\prime} .03($ or $5.0 \pm 1.0 \mathrm{pc})$ and $M_{\mathrm{NSC}}=(1.67 \pm 0.48) \times$ $10^{7} \mathrm{M}_{\odot}$, fully consistent with those derived from our radial stellarmass profile (see Table 6), as expected. As a consistency check, we also verified that the total mass of the stellar disc inferred here $\left[(1.3 \pm 0.4) \times 10^{10} \mathrm{M}_{\odot}\right.$; see Table 6] is in agreement with that of the two counterrotating stellar discs modelled by Coccato et al. (2013) $\left(\approx 1.5 \times 10^{10} \mathrm{M}_{\odot}\right.$; see Section 2$)$, on which our stellar-mass model is ultimately based (see Sections 4.2 and 4.3). Assuming this total stellar mass to be that of a discy bulge, the bulge then clearly belongs to the sub-M* category and has a stellar mass in agreement with those of other Milky Way-like targets.

We also compare the CMC size and mass inferred from the first Sérsic component (Table 6) to those derived from the central Gaussian of the ISM MGE model (Table 3), that are $M_{\mathrm{CMC}}=$ $5.2 \times 10^{6} \mathrm{M}_{\odot}$ and $r_{\mathrm{CMC}, \mathrm{e}}=0^{\prime \prime} .39$ (or $13.3 \mathrm{pc}$ ). The CMC mass and size derived from the double-Sérsic fit are thus in good agreement with those derived from the MGE approach, if a little smaller $(\approx 5$ per cent larger and 20 per cent smaller, respectively).

The CMC is co-spatial with the NSC, both being located at the centre of the more extended and fainter CND. The CMC is however more extended than the NSC (see Table 6), suggesting that radiation from the NSC is at least partially shielded by dust and $\mathrm{H}_{2}$. However, high-spatial resolution observations of other targets have often revealed central CO depressions or holes (e.g. Barth et al. 2016a, b; Davis et al. 2017; Boizelle et al. 2019; North et al. 2019; Smith et al. 2019; Nguyen et al. 2020). The high incidence of these holes may be caused by the true absence of molecular gas or by changing excitation conditions (Imanishi et al. 2018; Izumi et al. 2018 ), but most importantly the size of these holes is $\sim R_{\mathrm{SOI}}$, that is not spatially resolved by our ALMA observations of NGC 3593. We therefore cannot rule out the presence of such a hole at the centre of NGC 3593. Higher-angular resolution observations with $\theta_{\text {FWHM }} \lesssim \theta_{\text {ROI }}$ are required to better resolve the CMC, and thus establish whether it is a genuine CMC or instead harbours a central hole currently unnoticed due to beam smearing.

\section{2 $M_{\mathrm{BH}}-M_{\mathrm{NSC}}$ scaling relation}

Graham (2020) recently discussed a new correlation between $M_{\mathrm{BH}}$ and $M_{\mathrm{NSC}}$ in low-mass galaxies and UCDs, that combines the $M_{\mathrm{NSC}-}$ $M_{\text {bulge }}$ and $M_{\mathrm{BH}}-M_{\text {bulge }}$ (or equivalently $M_{\mathrm{NSC}}-\sigma_{\star}$ and $M_{\mathrm{BH}}-\sigma_{\star}$ ) correlations (e.g. Scott, Graham \& Schombert 2013; Graham 2016; Capuzzo-Dolcetta \& Tosta e Melo 2017; Davis, Graham \& Cameron 2019b; Sahu, Graham \& Davis 2019a,b):

$\log \left(\frac{M_{\mathrm{NSC}}}{\mathrm{M}_{\odot}}\right)=(0.38 \pm 0.06) \log \left(\frac{M_{\mathrm{BH}}}{10^{7.89} \mathrm{M}_{\odot}}\right)+(7.70 \pm 0.20)$.

This $M_{\mathrm{BH}}-M_{\mathrm{NSC}}$ scaling relation, shown in Fig. 8, works well to predict $M_{\mathrm{BH}}$ or $M_{\mathrm{NSC}}$ in nearby low-mass galaxies and UCDs if either of the two masses is known. All objects are well-studied and nearby, and thus harbour bona fide SMBHs and NSCs with reliably-measured masses. Only the low-mass lenticular galaxy NGC 5102 is an obvious

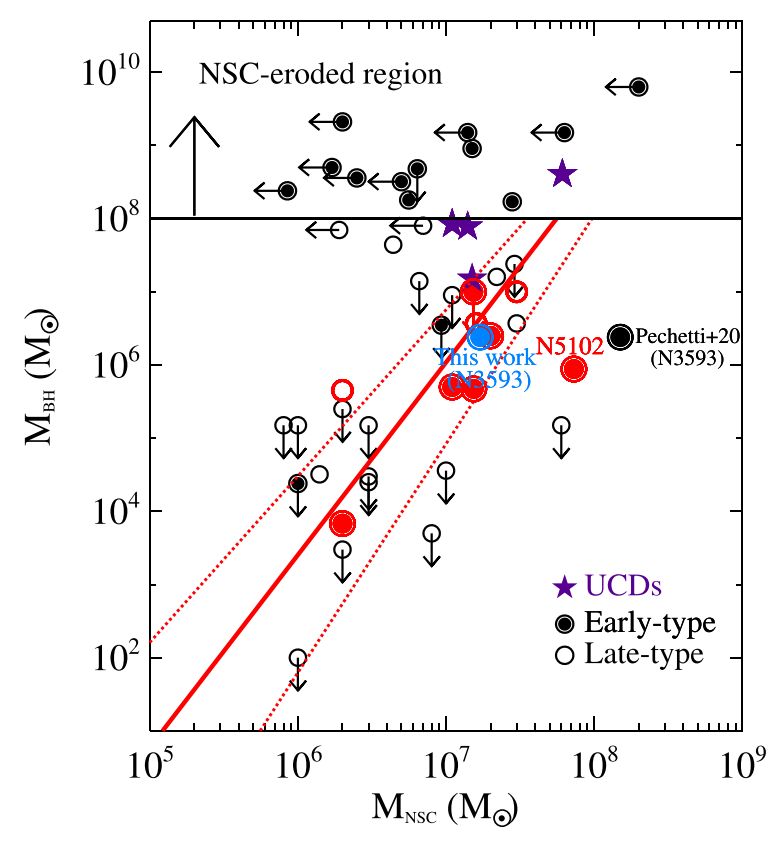

Figure 8. Graham's (2020) $M_{\mathrm{BH}}-M_{\mathrm{NSC}}$ scaling relation (red solid line) and its $1 \sigma$ uncertainties (red dotted lines). Purple stars and red circles show the inner stellar masses of four well-known UCDs (Ahn et al. 2017, 2018; Afanasiev et al. 2018; Voggel et al. 2018) and nine nearby NSCs (Graham \& Spitler 2009; Schödel, Merritt \& Eckart 2009; Lauer et al. 2012; Lyubenova et al. 2013; den Brok et al. 2015; Nguyen et al. 2017, 2018; Davis et al. 2020) with dynamical $M_{\mathrm{BH}}$ measurements, respectively. Black circles are NSCs taken from Neumayer \& Walcher (2012). Our own measurement in NGC 3593 is indicated by a cyan circle. Open and filled circles indicate lateand early-type galaxies, respectively. For galaxies with $M_{\mathrm{BH}} \gtrsim 10^{8} \mathrm{M}_{\odot}$, the NSCs erode at the expense of the BHs (Bekki \& Graham 2010; Neumayer et al. 2020).

outlier (Caldwell \& Bothun 1987; Davidge 2008; Nguyen et al. $2018,2019)$, owing to its very massive and extended NSC $\left(r_{\mathrm{NSC}, \mathrm{e}} \approx\right.$ $26 \mathrm{pc}, M_{\mathrm{NSC}}=7.3 \times 10^{7} \mathrm{M}_{\odot}$; Nguyen et al. 2018). An analogous inconsistency is found for NGC 3593 when we adopt the Pechetti et al.'s (2020) NSC mass. However, our own $M_{\mathrm{BH}}$ (Section 5.1) and $M_{\mathrm{NSC}}$ (Section 6.1) estimates are consistent with the Graham (2020) $M_{\mathrm{BH}}-M_{\mathrm{NSC}}$ scaling relation.

The truncation of the $M_{\mathrm{BH}}-M_{\mathrm{NSC}}$ scaling relation at $M_{\mathrm{BH}} \gtrsim 10^{8} \mathrm{M}_{\odot}$ (and resulting $M_{\mathrm{NSC}}$ upper limits; see Fig. 8) comes from the fact that while NSCs are common in low-mass galaxies $(\approx 75$ per cent of galaxies with $5 \times 10^{8} \lesssim M_{\star} \lesssim 10^{11} \mathrm{M}_{\odot}$ have NSCs; Böker et al. 2002; Côté et al. 2006; Seth et al. 2008a, b), more massive SMBHs in more massive galaxies (with $R_{\text {SOI }} \gtrsim 10 \mathrm{pc}$, larger than the NSCs themselves) start to erode their NSCs. Most of the UCDs with dynamically measured SMBHs are in this 'NSC-eroded region' and do not follow the $M_{\mathrm{BH}}-M_{\mathrm{NSC}}$ correlation.

The emerging $M_{\mathrm{BH}}-M_{\mathrm{NSC}}$ correlation in low-mass galaxies provides a new tool to predict the SMBH mass function at the low-mass end (e.g. Shankar et al. 2004; Graham \& Driver 2007; Vika et al. 2009; Kelly \& Shen 2013). This, in turn, will provide critical insight on the origin of UCDs as stellar remnant nuclei of threshed galaxies (e.g. Mieske et al. 2013; Seth et al. 2014), and will improve the accuracy of the predictions of the expected number of tidal disruption events (Stone \& Metzger 2016; Stone et al. 2017) and the SMBHSMBH and SMBH-stellar BH merging rates, all direct consequences of hierarchical galaxy formation (Voggel et al. 2019). 

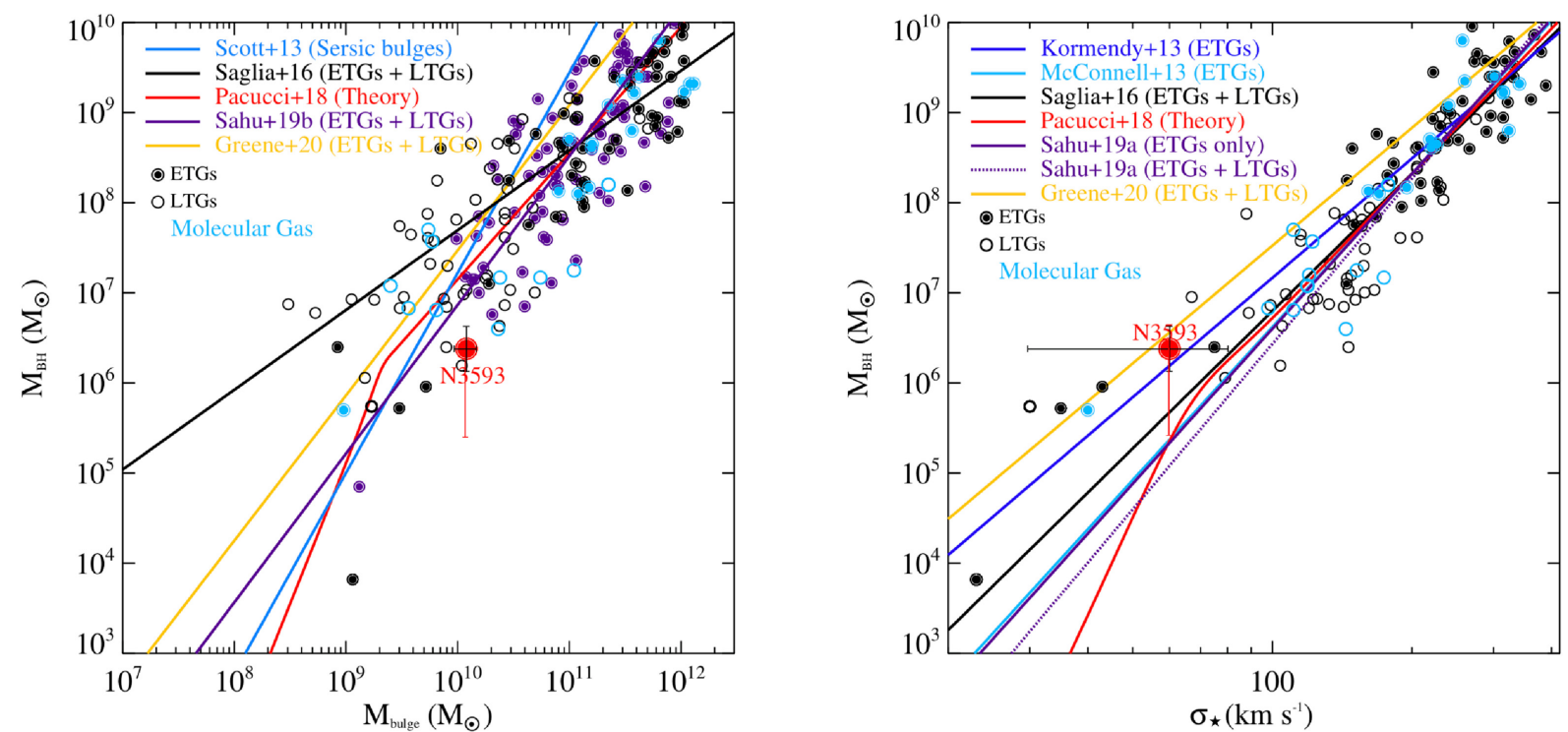

Figure 9. Our NGC $3593 M_{\mathrm{BH}}$ measurement (red filled circle) in the context of the $M_{\mathrm{BH}}-M_{\text {bulge }}$ (left-hand panel) and $M_{\mathrm{BH}}-\sigma_{\star}$ (right-hand panel) scaling relations. The error bars arising from the $3 \sigma$ statistical uncertainties of our default best-fitting model are plotted in black, while those that combine the systematic uncertainties associated with the stellar and/or ISM mass model are plotted in red. In total, 27 molecular-gas dynamical measurements using both ALMA (Onishi et al. 2015; Barth et al. 2016a, b; Davis et al. 2017, 2018, 2020; Boizelle et al. 2019, 2021; Combes et al. 2019; Nagai et al. 2019; North et al. 2019; Smith et al. 2019, 2021b; Nguyen et al. 2020, 2021; Cohn et al. 2021) and CARMA (Davis et al. 2013; Onishi et al. 2017) are plotted in cyan, with bulge masses taken from McConnell et al. (2013), Krajnović et al. (2013), Salo et al. (2015), Savorgnan et al. (2016), Nguyen et al. (2017, 2018, 2019), Sani et al. (2018). Other measurements taken from Saglia et al. (2016) and Sahu et al. (2019b) are plotted in black and purple, respectively. Early- and late-type galaxies are indicated with filled and open circles, respectively. The empirical scaling relations of Scott et al. (2013), Kormendy \& Ho (2013), McConnell et al. (2013), Saglia et al. (2016), Sahu et al. (2019a, 2019b), and Greene et al. (2020) are overlaid, colour-coded according to the legend. We also overlay the theoretical prediction of a bimodality from Pacucci et al. (2018; red line).

\section{DISCUSSION}

\section{1 $M_{\mathrm{BH}}$-galaxy properties scaling relations in the low-mass regime}

We now consider our NGC $3593 M_{\mathrm{BH}}$ measurement in the context of various $M_{\mathrm{BH}}-M_{\text {bulge }}$ (e.g. Scott et al. 2013; Saglia et al. 2016; Sahu et al. 2019b; Greene et al. 2020) and $M_{\mathrm{BH}^{-}} \sigma_{\star}$ (e.g. Kormendy \& Ho 2013; McConnell et al. 2013; Saglia et al. 2016; Sahu et al. 2019a,b; Greene et al. 2020) scaling relations, as shown in the leftand the right-hand panel of Fig. 9, respectively, and focusing on the low-mass regime. For the mass of the bulge of NGC 3593, we adopt the disc stellar mass listed in Table 6 (thus excluding the NSC; see also Section 6.1), assumed to be that of a discy bulge. For the stellar velocity dispersion of the bulge of NGC 3593, we adopt $\sigma_{\star} \approx$ $60 \mathrm{~km} \mathrm{~s}^{-1}$ from Bertola et al. (1996).

Our best-fitting NGC $3593 M_{\mathrm{BH}}$ inferred from molecular-gas dynamical modelling is fully consistent with the empirical $M_{\mathrm{BH}}-$ $M_{\text {bulge }}$ correlation of galaxies with cored central surface-brightness profiles (i.e. bulges) from Scott et al. (2013), the empirical $M_{\mathrm{BH}^{-}}$ $M_{\text {bulge }}$ correlation of both ETGs and LTGs from Sahu et al. (2019b), and the theoretical $M_{\mathrm{BH}}-M_{\text {bulge }}$ scaling relation of Pacucci et al. (2018). It is, however, offset negatively by about half an order of magnitude from the correlations of Saglia et al. (2016) and Greene et al. (2020). Our $M_{\mathrm{BH}}$ measurement is also offset negatively by about one order of magnitude from the correlations of Kormendy \& Ho (2013) and McConnell et al. (2013), that are however not shown in Fig. 9 as they are constructed primarily from highmass galaxies with cuspy profiles (i.e. galaxies without central cores).
With a mass of $\approx 2.4 \times 10^{6} \mathrm{M}_{\odot}$, the SMBH of NGC 3593 is at the low-mass end of the Combes et al. (2019) sample, and it is the second lowest $M_{\mathrm{BH}}$ measured so far using molecular gas kinematics (the lowest currently being $M_{\mathrm{BH}} \approx 5 \times 10^{5} \mathrm{M}_{\odot}$ in NGC 404; Davis et al. 2020). Our measurement of the NGC 3593 SMBH mass also fills in a gap for ETGs between low-mass $\left(M_{\text {bulge }} \leq 5 \times 10^{10} \mathrm{M}_{\odot}\right.$ and $M_{\mathrm{BH}} \leq 10^{7} \mathrm{M}_{\odot}$; e.g. Nguyen et al. 2018) and high-mass ( $M_{\text {bulge }}>$ $5 \times 10^{10} \mathrm{M}_{\odot}$ and $M_{\mathrm{BH}}>10^{7} \mathrm{M}_{\odot}$ ) targets. It is also becoming clear that the $M_{\mathrm{BH}}-M_{\text {bulge }}$ correlation has a break at a specific 'transition' mass (Pacucci et al. 2017; Nguyen et al. 2019). More measurements

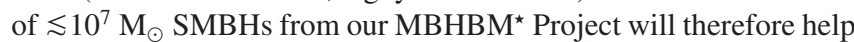
to confirm this break and pinpoint the exact transition mass, while simultaneously helping to calibrate $M_{\mathrm{BH}^{-}}$galaxy scaling relations across the higher galaxy mass range $\left(M_{\text {bulge }} \gtrsim 10^{11} \mathrm{M}_{\odot}\right)$.

Concerning the $M_{\mathrm{BH}}-\sigma_{\star}$ correlation, the mass of the SMBH of NGC 3593 is favouring the Greene et al. (2020) correlation over others (Kormendy \& Ho 2013; McConnell et al. 2013; Saglia et al. 2016; Pacucci et al. 2018; Sahu et al. 2019a,b). Indeed, it is offset positively by one half to one order of magnitude from these correlations, similarly to other measurements at low $\sigma_{\star}$ by den Brok et al. (2015) and Nguyen et al. (2018, 2019). The reason for these systematic offsets is currently unknown. However, higherspatial resolution observations of the stars and warm ionized gas with James Webb Space Telescope, and of the molecular gas (e.g. CO; Nguyen et al. 2020, 2021) and atomic gas (e.g. [CI](1-0); Nguyen et al. 2021) with ALMA, that can probe the kinematics closer to or within the predicted SOIs, will help shed light on this issue before the era of extremely large ground-based optical telescopes and the next phase of ALMA itself. 


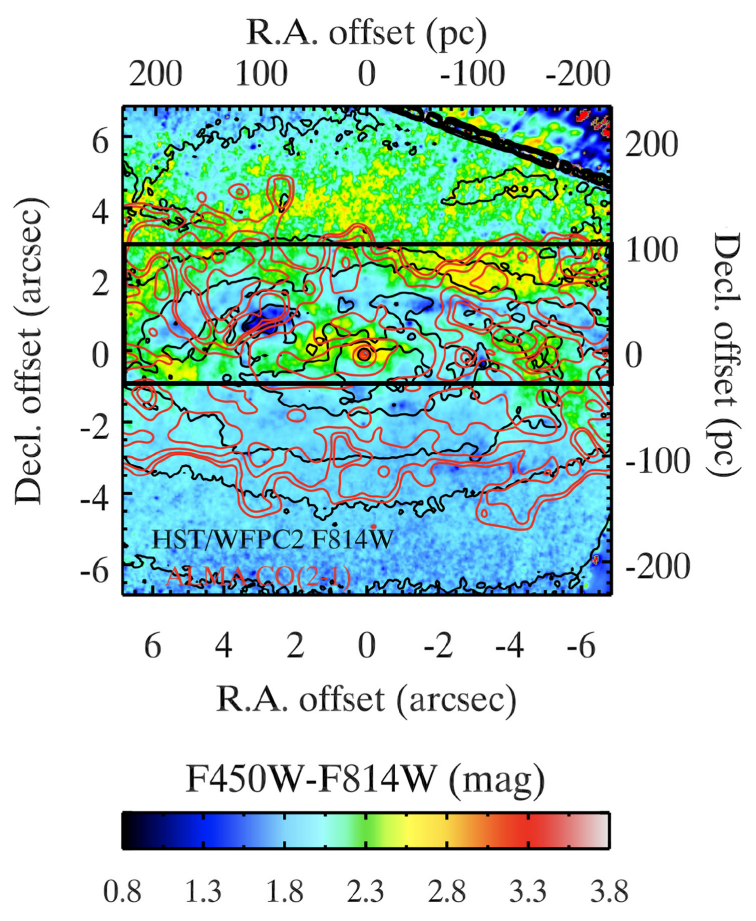

Figure 10. HST WFPC2 F450W-F814W colour map of the nucleus of NGC 3593 (see also Panel A of Fig. B5 in Appendix B), overlaid with ${ }^{12} \mathrm{CO}(2-$ 1) (red) and $F 814 \mathrm{~W}$ (black) iso-intensity contours. The black rectangle indicates the region with co-spatial molecular gas and dust extinction discussed in the text.

\subsection{Extended morphology: inflow or outflow?}

As discussed previously, the morphology of the molecular gas in the CND of NGC 3593 suggests the presence of a nuclear disc with a two-arm spiral pattern, i.e. bi-symmetric arms extending from the centre to a ring-like structure farther out (see panels A and B of Fig. B1 in Appendix B). Such features may be associated with shocks, inflows/outflows and/or filaments in the molecular gas disc, and they have been observed at both millimetre/sub-millimetre wavelengths with ALMA (cold molecular gas; e.g. Combes et al. 2013, 2014; Espada et al. 2017) and in the NIR with telescopes such as VLT and Gemini (hot $\mathrm{H}_{2}$; e.g. Riffel, Storchi-Bergmann \& Winge 2013; Davies et al. 2014; Diniz et al. 2015).

The high-intensity regions visible in the bi-symmetric arms and the extended disc could be caused by the compression of gas along the two-arm spiral pattern. Such features (nuclear spirals and rings) are believed to form in the centres of non-axisymmetric potentials, that cause the gas to lose angular momentum and shock (e.g. Maciejewski \& Sparke 2000; Emsellem et al. 2001; Shlosman 2001; Maciejewski 2004a, b; Shlosman 2005; Fathi et al. 2007). The gas then flows into the nucleus along the shocks, thus forming the observed nuclear spirals and rings (e.g. Fathi et al. 2006; Casasola et al. 2011; Combes et al. 2013, 2014). However, there is no evidence of a bar in the optical morphology (Fig. 1) or the F450W-F814W colour map (Panel A of Fig. B5 in Appendix B) of NGC 3593. Thus, the formation of the observed two-arm spiral pattern in NGC 3593 is unlikely to be driven by a barred potential.

Alternatively, gas from beyond the ring may fall toward the galaxy centre via dynamical friction, potentially also giving rise to a nonaxisymmetric potential and thus to the formation of a two-arm spiral. In this scenario, some of the angular momentum from the infalling gas is transferred to the molecular gas disc, creating a shock front and the clumpy high surface brightness regions (e.g. Malkan, Gorjian \& Tam 1998). The spatial coincidence of these high-intensity, high-velocity dispersion and high-velocity residual regions (see e.g. Panels DL of Fig. B8 in Appendix B and Panels D-F of Fig. 4) supports this scenario of externally accreting material, that has not yet fully settled into the host galaxy potential to form a regular and relaxed disc. Supporting evidence in the form of larger-scale filaments and/or warps is however lacking. Future observations with both shorter $\left(\theta_{\text {FWHM }} \gtrsim 1\right.$ arcsec $)$ and longer $\left(\theta_{\text {FWHM }} \lesssim 0^{\prime \prime}\right.$. 1$)$ baselines would be valuable to fully unravel the origin of the nuclear two-arm spiral pattern.

Similarly, both theoretical and observational studies have discussed the role of nuclear spiral arms as bridges to transport molecular gas from kpc scales to nuclei via the removal of angular momentum, possibly also feeding central SMBHs (e.g. Wada \& Norman 2002; Maciejewski 2004b, a; Fathi et al. 2006, 2011, 2013; Casasola et al. 2008; Hopkins \& Quataert 2010; van de Ven \& Fathi 2010; Combes et al. 2013, 2014). On the other hand, nuclear spiral arms can perturb the ambient gas, creating turbulence. This can lead to shock-driven streaming motions and outflows (van de Ven \& Fathi 2010), which could explain the features seen in the residual velocity map of the CND of NGC 3593 (Panel F of Fig. B8 in Appendix B and 4).

Although our ALMA data reveal a two-arm spiral structure in the nucleus of NGC 3593, it is weak/flocculent so its dynamical impact on the circumnuclear region remains unclear. We are therefore unable to firmly conclude that the CND non-circular motions identified through our dynamical modelling can only be explained by either inflows or outflows associated with the observed two-arm spiral structure.

\subsection{Origin of the molecular gas: external accretion?}

The association between the nuclear dust lanes seen in HST optical images and the morphology of the ${ }^{12} \mathrm{CO}(2-1)$ emission detected in the CND with ALMA confirms the spatial coincidence of dust and molecular gas (for ETGs, see e.g. Prandoni et al. 2007, 2010; Young et al. 2011; Alatalo et al. 2013; Nyland et al. 2017). Fig. 10 shows the F450W-F814W colour image overlaid with the ${ }^{12} \mathrm{CO}(2-1)$ and $F 814 \mathrm{~W}$ iso-intensity contours. While dust and molecular gas are cospatial within an $\approx 4 \times 14 \operatorname{arcsec}^{2}\left(\approx 140 \times 490 \mathrm{pc}^{2}\right)$ region indicated by a black rectangle in Fig. 10, the faint molecular gas south of the major axis does not trace obvious dust structures. In addition, there is significant dust extinction beyond the detected ${ }^{12} \mathrm{CO}(2-1)$ emission to the north of the major axis ( $>4 \operatorname{arcsec}$ or $140 \mathrm{pc}$ ), but within the ALMA antennae primary beam (see panel A of Fig. B1 in Appendix $\mathrm{B})$, suggesting the presence of more but fainter molecular gas below our detection threshold $\left(\approx 1 \mathrm{mJy}\right.$ beam ${ }^{-1}$ per $10 \mathrm{~km} \mathrm{~s}^{-1}$ binned channel).

Despite its lenticular type, NGC 3593 harbours a large amount of not only molecular gas $\left(M_{\mathrm{H}_{2}}=(2.8 \pm 1.2) \times 10^{8} \mathrm{M}_{\odot}\right.$; Section 4.2) but also atomic gas $\left(M_{\mathrm{HI}}=1.3 \times 10^{8} \mathrm{M}_{\odot}\right.$; Pogge \& Eskridge 1993), suggesting a recent replenishment of its neutral gas reservoir (Young et al. 2014; Babyk et al. 2019; Davis et al. 2019a). The origin of neutral gas in ETGs can be either internal (stellar mass loss or gas remaining from the galaxy formation process; e.g. Davis \& Young 2019) or external (most likely accretion during minor mergers of gas-rich dwarfs; see e.g. Storchi-Bergmann \& Schnorr-Müller 2019 for a review).

Given the absence of a stellar bar that could funnel gas to the centre (see Section 7.2), and as the galaxy also hosts lots of young stars that quickly return gas to the ISM (e.g. Davis et al. 2016; Davis \& Young 
2019), stellar mass loss appears to be a viable scenario in NGC 3593. As the younger of the two stellar disc is counterrotating, this gas would itself also be naturally counterrotating. Having said that, Davis et al. (2019a) developed a toy model including a variety of merger (e.g. Couto et al. 2013; Fischer et al. 2015; Riffel, Storchi-Bergmann \& Riffel 2015; Couto et al. 2016; Couto, Storchi-Bergmann \& Schnorr-Müller 2017; Raimundo et al. 2017) and feedback processes to estimate the gas-rich merger rate and thus the mean gas fraction of ETGs in the local universe. They found that only 25 per cent of all low-redshift ETGs harbouring cold gas in clusters are the remnants of transformed LTGs. The remaining 75 per cent could be the results of gas-rich mergers.

Both internal (stellar mass loss) and external (gas-rich minor merger) mechanisms are thus likely to simultaneously take place in NGC 3593. The former is evidenced by the intensive ongoing star formation within the CND, and by the galaxy's early state of transformation from a spiral into an ETG (as a lenticular galaxy). The latter is evidenced by the co-spatial but counterrotating stellar, ionized-gas and molecular-gas discs, as misalignment of the angular momenta of cold gas and stars is expected in a merger (e.g. Young 2002; Young, Bureau \& Cappellari 2008; Crocker et al. 2011; Davis et al. 2011; Lagos et al. 2015).

\section{CONCLUSIONS}

We have presented new ALMA observations of ${ }^{12} \mathrm{CO}(2-1)$ emission in the nucleus of the lenticular galaxy NGC 3593, that in combination with HST optical images and dynamical modelling reveal the presence of a central SMBH. We summarize our results as follows:

(i) NGC 3593 hosts a highly inclined $\left(i \approx 75^{\circ}\right){ }^{12} \mathrm{CO}(2-1) \mathrm{CND}$ extending over a region of $\approx 30 \times 10 \operatorname{arcsec}^{2}\left(\approx 1050 \times 350 \mathrm{pc}^{2}\right)$ elongated along the galaxy major axis, with a two-arm/bi-symmetric spiral pattern surrounded by a ring-like structure of $\approx 10 \operatorname{arcsec}$ $(\approx 350 \mathrm{pc})$ radius. The molecular gas distribution and kinematics reveal the CND to be largely dynamically settled, and coincident and co-rotating with the known ionized-gas and secondary stellar disc (all counterrotating with respect to the primary stellar disc).

(ii) The ${ }^{12} \mathrm{CO}(2-1)$ kinematics beyond the nucleus allow us to constrain the outer mass (i.e. stellar and ISM mass surface density) profile of the galaxy accurately, useful to appropriately scale the inner stellar-mass profile, that itself has a large impact on our dynamical $M_{\mathrm{BH}}$ measurement.

(iii) Our default best-fitting dynamical model requires a central SMBH mass $M_{\mathrm{BH}}=2.40_{-1.05}^{+1.87} \times 10^{6} \mathrm{M}_{\odot}$ and a stellar-mass scaling factor $\Gamma=0.89_{-0.03}^{+0.06}$, suggesting that our stellar-mass model and choice of a Chabrier IMF are reasonable (statistical uncertainties only, at the $3 \sigma$ level). Considering all potential systematic uncertainties associated with the stellar and/or ISM mass model, the SMBH must have a mass in the range $3.0 \times 10^{5}-4.3 \times 10^{6} \mathrm{M}_{\odot}$.

(iv) The inferred SMBH mass is consistent with the empirical $M_{\mathrm{BH}}-M_{\text {bulge }}$ correlation of cored galaxies (Scott et al. 2013) and the recent compilation of Sahu et al. (2019b), but it is almost one order of magnitude below the Kormendy \& Ho (2013) and Saglia et al. (2016) scaling relations for more massive galaxies/BH. Regarding the $M_{\mathrm{BH}}-\sigma_{\star}$ correlation, NGC 3593 is consistent with the correlation of Greene et al. (2020), but it is about half an order of magnitude above that of McConnell et al. (2013) and one order of magnitude above those of Saglia et al. (2016) and Sahu et al. (2019b).

(v) Our accurate stellar-mass model yields improved constraints on the NSC, with a total stellar mass $M_{\mathrm{NSC}}$ approximately 10 times smaller than that derived purely photometrically by Pechetti et al.
(2020), thus making our new $M_{\mathrm{BH}}$ and $M_{\mathrm{NSC}}$ consistent with the recent $M_{\mathrm{BH}}-M_{\mathrm{NSC}}$ scaling relation of Graham (2020).

(vi) We detect a CMC co-spatial with the NSC and well described by a Sérsic profile with an effective radius $r_{\mathrm{CMC}, \mathrm{e}}=11.2 \pm 2.8 \mathrm{pc}$, a Sérsic index $n_{\mathrm{CMC}}=1.1 \pm 0.1$ and a total ISM mass $M_{\mathrm{CMC}}=$ $(5.4 \pm 1.2) \times 10^{6} \mathrm{M}_{\odot}$.

(vii) We have identified a few regions of non-circular gas motions in the ${ }^{12} \mathrm{CO}(2-1) \mathrm{CND}$, likely associated with the two-arm spiral pattern, but it is unclear whether they are leading to any outflow or inflow. The two-arm spiral could have been formed by gas accretion from the outer gas reservoirs $(r \gtrsim 15 \operatorname{arcsec}$ or $525 \mathrm{pc}$ ) via dynamical friction.

(viii) The significant molecular (and atomic) gas reservoir in a lenticular galaxy like NGC 3593, counterrotating with respect to the primary stellar disc, suggests a primarily external gas origin via a gas-rich minor merger, possibly associated with internal stellar mass loss in the younger/more recent stellar component.

\section{DATA AVAIBILITY}

The data underlying this article will be shared on reasonable request to the corresponding author. Alternatively, the pipeline calibrated ALMA data is available from the archive with the project code 2017.1.00964.S

\section{ACKNOWLEDGEMENTS}

The authors would like to thank the anonymous referee for their careful reading and useful comments, that helped to improve the paper greatly. DDN would like to thank the International University - Vietnam National University in Ho Chi Minh City, the National Astrononical Observatory of Japan (NAOJ) and the National Institute of Natural Sciences (NINS) for supporting this work. MB was supported by the consolidated grants Astrophysics at Oxford ST/H002456/1 and ST/K00106X/1 from the United Kingdom Research Councils. ST acknowledges funding from the European Research Council (ERC) under the European Union's Horizon 2020 research and innovation programme under grant agreement No 724857 (Consolidator Grant ArcheoDyn). TAD acknowledges support from Science and Technology Facilities Council (STFC) grant ST/S00033X/1. MC expresses his gratitude for a Royal Society University Research Fellowship (RSURF). TI and SB are supported by Japan Society for the Promotion of Science (JSPS) KAKENHI grant number 17K14247 and 19J00892, respectively. Basic research in radio astronomy at the US Naval Research Laboratory is supported by 6.1 Base Funding. The authors also thank Mark D. Smith of the University of Oxford for his enlightening discussions on using the SkySampler tool.

This paper makes use of the following ALMA data: ADS/JAO.ALMA\#2017.1.00964.S. ALMA is a partnership of ESO (representing its member states), NSF (USA) and NINS (Japan), together with NRC (Canada) and NSC and ASIAA (Taiwan) and KASI (Republic of Korea), in cooperation with the Republic of Chile. The Joint ALMA Observatory is operated by ESO, AUI/NRAO, and NAOJ. The National Radio Astronomy Observatory is a facility of the National Science Foundation operated under cooperative agreement by Associated Universities, Inc. We thank the ALMA operators and staff and the ALMA help desk for diligent feedback and invaluable assistance during the processing of these data.

Facilities: ALMA and HST WPFC2.

Software: IDL, CASA, Python, astropy, emcee, KinMS, MgeFit, IRAF, and Kinemetry. 


\section{REFERENCES}

Abolfathi B. et al., 2018, ApJS, 235, 42

Afanasiev A. V. et al., 2018, MNRAS, 477, 4856

Ahn C. P. et al., 2017, ApJ, 839, 72

Ahn C. P. et al., 2018, ApJ, 858, 102

Alatalo K. et al., 2013, MNRAS, 432, 1796

Babyk I. V., McNamara B. R., Tamhane P. D., Nulsen P. E. J., Russell H. R., Edge A. C., 2019, ApJ, 887, 149

Balcells M., González A. C., 1998, ApJ, 505, L109

Baldassare V. F., Reines A. E., Gallo E., Greene J. E., 2015, ApJ, 809, L14

Baldassare V. F., Dickey C., Geha M., Reines A. E., 2020, ApJ, 898, L3

Baldry I. K. et al., 2012, MNRAS, 421, 621

Barth A. J., Ho L. C., Rutledge R. E., Sargent W. L. W., 2004, ApJ, 607, 90

Barth A. J., Boizelle B. D., Darling J., Baker A. J., Buote D. A., Ho L. C., Walsh J. L., 2016a, ApJ, 822, L28

Barth A. J., Darling J., Baker A. J., Boizelle B. D., Buote D. A., Ho L. C., Walsh J. L., 2016b, ApJ, 823, 51

Bekki K., Graham A. W., 2010, ApJ, 714, L313

Bertola F., Cinzano P., Corsini E. M., Pizzella A., Persic M., Salucci P., 1996, ApJ, 458, L67

Bigiel F., Leroy A., Walter F., Brinks E., de Blok W. J. G., Madore B., Thornley M. D., 2008, AJ, 136, 2846

Binney J., Tremaine S., 1987, Galactic Dynamics. Cambridge Univ. Press, Cambridge

Bois M. et al., 2011, MNRAS, 416, 1654

Boizelle B. D., Barth A. J., Walsh J. L., Buote D. A., Baker A. J., Darling J., Ho L. C., 2019, ApJ, 881, 10

Boizelle B. D. et al., 2021, ApJ, 908, 19

Böker T., Laine S., van der Marel R. P., Sarzi M., Rix H.-W., Ho L. C., Shields J. C., 2002, AJ, 123, 1389

Bolatto A. D., Wolfire M., Leroy A. K., 2013, ARA\&A, 51, 207

Bonoli S., Mayer L., Callegari S., 2014, MNRAS, 437, 1576

Buta R. J., Corwin H. G., Odewahn S. C., 2007, The de Vaucouleurs Atlas of Galaxies. Cambridge Univ. Press, Cambridge

Calabrese E. et al., 2017, Phys. Rev. D, 95, 063525

Caldwell N., Bothun G. D., 1987, AJ, 94, 1126

Cappellari M., 2002, MNRAS, 333, 400

Cappellari M., 2008, MNRAS, 390, 71

Capuzzo-Dolcetta R., Tosta e Melo I., 2017, MNRAS, 472, 4013

Cardelli J. A., Clayton G. C., Mathis J. S., 1989, ApJ, 345, 245

Casasola V., Combes F., García-Burillo S., Hunt L. K., Léon S., Baker A. J., 2008, A\&A, 490, 61

Casasola V., Hunt L. K., Combes F., García-Burillo S., Neri R., 2011, A\&A, 527, A92

Cautun M. et al., 2020, MNRAS, 494, 4291

Charlot S., Fall S. M., 2000, ApJ, 539, 718

Chilingarian I. V., Katkov I. Y., Zolotukhin I. Y., Grishin K. A., Beletsky Y., Boutsia K., Osip D. J., 2018, ApJ, 863, 1

Coccato L., Morelli L., Pizzella A., Corsini E. M., Buson L. M., Dalla Bontà E., 2013, A\&A, 549, A3

Cohn J. H. et al., 2021, ApJ, 919, 77

Combes F. et al., 2013, A\&A, 558, A124

Combes F. et al., 2014, A\&A, 565, A97

Combes F. et al., 2019, A\&A, 623, A79

Corsini E. M., Pizzella A., Funes J. G., Vega Beltran J. C., Bertola F., 1998, A\&A, 337, 80

Corwin Harold G. J., Buta R. J., de Vaucouleurs G., 1994, AJ, 108, 2128

Côté P. et al., 2006, ApJS, 165, 57

Couto G. S., Storchi-Bergmann T., Axon D. J., Robinson A., Kharb P., Riffel R. A., 2013, MNRAS, 435, 2982

Couto G. S., Storchi-Bergmann T., Robinson A., Riffel R. A., Kharb P., Lena D., Schnorr-Müller A., 2016, MNRAS, 458, 855

Couto G. S., Storchi-Bergmann T., Schnorr-Müller A., 2017, MNRAS, 469, 1573

Cresci G., Vanzi L., Sauvage M., Santangelo G., van der Werf P., 2010, A\&A, 520, A 82
Crocker A. F., Bureau M., Young L. M., Combes F., 2011, MNRAS, 410, 1197

Dame T. M., 2011, preprint (arXiv:1101.1499)

Dame T. M., Hartmann D., Thaddeus P., 2001, ApJ, 547, 792

Davidge T. J., 2008, AJ, 135, 1636

Davies R. I. et al., 2014, ApJ, 792, 101

Davis T. A., 2014, MNRAS, 443, 911

Davis T. A., Young L. M., 2019, MNRAS, 489, L108

Davis T. A. et al., 2011, MNRAS, 417, 882

Davis T. A., Bureau M., Cappellari M., Sarzi M., Blitz L., 2013, Nature, 494, 328

Davis T. A., Greene J., Ma C.-P., Pandya V., Blakeslee J. P., McConnell N., Thomas J., 2016, MNRAS, 455, 214

Davis T. A., Bureau M., Onishi K., Cappellari M., Iguchi S., Sarzi M., 2017, MNRAS, 468, 4675

Davis T. A. et al., 2018, MNRAS, 473, 3818

Davis T. A., Greene J. E., Ma C.-P., Blakeslee J. P., Dawson J. M., Pandya V., Veale M., Zabel N., 2019a, MNRAS, 486, 1404

Davis T. A. et al., 2020, MNRAS, 496, 4061

Davis B. L., Graham A. W., Cameron E., 2019b, ApJ, 873, 85

de Vaucouleurs G., de Vaucouleurs A., Corwin H. G., Jr, Buta R. J., Paturel G., Fouqué P., 1991, Third Reference Catalogue of Bright Galaxies. Volume I: Explanations and references. Volume II: Data for galaxies between $0^{h}$ and $12^{h}$. Volume III: Data for galaxies between $12^{h}$ and $24^{h}$. Springer, New York, NY

den Brok M. et al., 2015, ApJ, 809, 101

Desroches L.-B., Greene J. E., Ho L. C., 2009, ApJ, 698, 1515

Diniz M. R., Riffel R. A., Storchi-Bergmann T., Winge C., 2015, MNRAS, 453, 1727

Dong X.-B., Ho L. C., Yuan W., Wang T.-G., Fan X., Zhou H., Jiang N., 2012, ApJ, 755, 167

Draine B. T., Lee H. M., 1984, ApJ, 285, 89

Eliche-Moral M. C., González-García A. C., Balcells M., Aguerri J. A. L., Gallego J., Zamorano J., Prieto M., 2011, A\&A, 533, A104

Emsellem E., Monnet G., Bacon R., 1994, A\&A, 285, 723

Emsellem E., Greusard D., Combes F., Friedli D., Leon S., Pécontal E., Wozniak H., 2001, A\&A, 368, 52

Espada D. et al., 2017, ApJ, 843, 136

Fathi K., Storchi-Bergmann T., Riffel R. A., Winge C., Axon D. J., Robinson A., Capetti A., Marconi A., 2006, ApJ, 641, L25

Fathi K., van de Ven G., Peletier R., Emsellem E., Falcón-Barroso J., Cappellari M., de Zeeuw T., 2007, Astrophys. Space Sci. Proce., 3, 125

Fathi K., Axon D. J., Storchi-Bergmann T., Kharb P., Robinson A., Marconi A., Maciejewski W., Capetti A., 2011, ApJ, 736, 77

Fathi K. et al., 2013, ApJ, 770, L27

Ferrarese L., Merritt D., 2000, ApJ, 539, L9

Fich M., 1993, ApJS, 86, 475

Fischer T. C., Crenshaw D. M., Kraemer S. B., Schmitt H. R., StorchiBergmann T., Riffel R. A., 2015, ApJ, 799, 234

Foreman-Mackey D., Hogg D. W., Lang D., Goodman J., 2013, Publ. Astron. Soc. Pac, 125, 306

Gallo E., Treu T., Jacob J., Woo J.-H., Marshall P. J., Antonucci R., 2008, ApJ, 680, 154

Gallo E., Treu T., Marshall P. J., Woo J.-H., Leipski C., Antonucci R., 2010, ApJ, 714, 25

García-Burillo S., Sempere M. J., Combes F., Hunt L. K., Neri R., 2000, A\&A, 363, 869

Gebhardt K. et al., 2000, ApJ, 539, L13

Goodman J., Weare J., 2010, Commun. Appl. Math. Comput. Sci., 5, 65

Graham M. T. et al., 2018, MNRAS, 477, 4711

Graham A. W., 2016, in Meiron Y., Li S., Liu F. K., Spurzem R., eds, IAU Symp. Vol. 312, Star Clusters and Black Holes in Galaxies across Cosmic Time. Kluwer, Dordrecht, p. 269

Graham A. W., 2020, MNRAS, 492, 3263

Graham A. W., Driver S. P., 2007, MNRAS, 380, L15

Graham A. W., Spitler L. R., 2009, MNRAS, 397, 2148

Greene J. E., 2012, Nat. Commun., 3, 1304

Greene J. E., Ho L. C., 2007, ApJ, 670, 92 
Greene J. E., Strader J., Ho L. C., 2020, ARA\&A, 58, 257

Haemmerlé L., Mayer L., Klessen R. S., Hosokawa T., Madau P., Bromm V., 2020, Space Sci. Rev., 216, 48

Häring N., Rix H.-W., 2004, ApJ, 604, L89

Hildebrand R. H., 1983, QJRAS, 24, 267

Ho L. C., Filippenko A. V., Sargent W. L. W., 1997, ApJS, 112, 315

Holtzman J. A. et al., 1995, Publ. Astron. Soc. Pac, 107, 156

Hopkins P. F., Quataert E., 2010, MNRAS, 405, L41

Hunter D. A., Thronson Harley A. J., Casey S., Harper D. A., 1989, ApJ, 341, 697

Imanishi M., Nakanishi K., Izumi T., Wada K., 2018, ApJ, 853, L25

Inayoshi K., Visbal E., Haiman Z., 2020, ARA\&A, 58, 27

Izumi T., Wada K., Fukushige R., Hamamura S., Kohno K., 2018, ApJ, 867, 48

Jedrzejewski R. I., 1987, MNRAS, 226, 747

Jesseit R., Naab T., Peletier R. F., Burkert A., 2007, MNRAS, 376, 997

Kacharov N., Neumayer N., Seth A. C., Cappellari M., McDermid R., Walcher C. J., Böker T., 2018, MNRAS, 480, 1973

Karachentsev I. D., Karachentseva V. E., Huchtmeier W. K., Makarov D. I., 2004, AJ, 127, 2031

Kelly B. C., Shen Y., 2013, ApJ, 764, 45

Kormendy J., Ho L. C., 2013, ARA\&A, 51, 511

Kormendy J., Richstone D., 1995, ARA\&A, 33, 581

Krajnović D. et al., 2013, MNRAS, 432, 1768

Krajnović D. et al., 2018, MNRAS, 477, 3030

Krajnović D., Cappellari M., de Zeeuw P. T., Copin Y., 2006, MNRAS, 366, 787

Krist J., 1995, in Shaw R. A., Payne H. E., Hayes J. J. E., eds, ASP Conf. Ser. Vol. 77, Astronomical Data Analysis Software and Systems IV. Astron. Soc. Pac., San Francisco, p. 349

Krist J. E., Hook R. N., Stoehr F., 2011, in Kahan M. A., ed., Proc. SPIE Conf. Ser. Vol. 8127, Optical Modeling and Performance Predictions V. SPIE, Bellingham, p. 81270J

Kuno N., Nishiyama K., Nakai N., Sorai K., Vila-Vilaró B., Handa T., 2000, Publ. Astron. Soc. Japan, 52, 775

Kuno N. et al., 2007, Publ. Astron. Soc. Japan, 59, 117

Kuo C. Y. et al., 2011, ApJ, 727, 20

Lagos C. d. P., Padilla N. D., Davis T. A., Lacey C. G., Baugh C. M., GonzalezPerez V., Zwaan M. A., Contreras S., 2015, MNRAS, 448, 1271

Lauer T. R., Bender R., Kormendy J., Rosenfield P., Green R. F., 2012, ApJ, 745,121

Lo K. Y., 2005, ARA\&A, 43, 625

Lodato G., Natarajan P., 2006, MNRAS, 371, 1813

Lyubenova M. et al., 2013, MNRAS, 431, 3364

McMullin J. P., Waters B., Schiebel D., Young W., Golap K., 2007, in Shaw R. A., Hill F., Bell D. J., eds, ASP Conf. Ser. Vol. 376, Astronomical Data Analysis Software and Systems XVI. Astron. Soc. Pac., San Francisco, p. 127

McConnell N. J., Chen S.-F. S., Ma C.-P., Greene J. E., Lauer T. R., Gebhardt K., 2013, ApJ, 768, L21

Maciejewski W., 2004a, MNRAS, 354, 883

Maciejewski W., 2004b, MNRAS, 354, 892

Maciejewski W., Sparke L. S., 2000, MNRAS, 313, 745

Magorrian J. et al., 1998, AJ, 115, 2285

Maksym W. P., Ulmer M. P., Eracleous M. C., Guennou L., Ho L. C., 2013, MNRAS, 435, 1904

Malkan M. A., Gorjian V., Tam R., 1998, ApJS, 117, 25

Marconi A., Hunt L. K., 2003, ApJ, 589, L21

Markwardt C. B., 2009, in Bohlender D. A., Durand D., Dowler P., eds, ASP Conf. Ser. Vol. 411, Astronomical Data Analysis Software and Systems XVIII. Astron Soc. Pac., San Francisco, p. 251

Martínez-García E. E., González-Lópezlira R. A., Magris C. G., Bruzual A. G., 2017, ApJ, 835, 93

Mieske S., Frank M. J., Baumgardt H., Lützgendorf N., Neumayer N., Hilker M., 2013, A\&A, 558, A14

Miller B. P., Gallo E., Greene J. E., Kelly B. C., Treu T., Woo J.-H., Baldassare V., 2015, ApJ, 799, 98

Mitzkus M., Cappellari M., Walcher C. J., 2017, MNRAS, 464, 4789
Miyoshi M., Moran J., Herrnstein J., Greenhill L., Nakai N., Diamond P., Inoue M., 1995, Nature, 373, 127

Moran E. C., Shahinyan K., Sugarman H. R., Vélez D. O., Eracleous M., 2014, AJ, 148, 136

Moura-Santos E., Carvalho F. C., Penna-Lima M., Novaes C. P., Wuensche C. A., 2016, ApJ, 826, 121

Nagai H. et al., 2019, ApJ, 883, 193

Neumayer N., Walcher C. J., 2012, Adv. Astron., 2012, 709038

Neumayer N., Cappellari M., Reunanen J., Rix H.-W., van der Werf P. P., de Zeeuw P. T., Davies R. I., 2007, ApJ, 671, 1329

Neumayer N., Seth A., Boeker T., 2020, Astron. Astrophys. Rev., 28, 4

Nguyen D. D., 2017, preprint (arXiv:1712.02470)

Nguyen D. D., Seth A. C., Reines A. E., den Brok M., Sand D., McLeod B., 2014, ApJ, 794, 34

Nguyen D. D. et al., 2017, ApJ, 836, 237

Nguyen D. D. et al., 2018, ApJ, 858, 118

Nguyen D. D. et al., 2019, ApJ, 872, 104

Nguyen D. D. et al., 2020, ApJ, 892, 68

Nguyen D. D. et al., 2021, MNRAS, 504, 4123

Nguyen D., 2019, ALMA2019: Science Results and Cross-Facility Synergies. p. 106

North E. V. et al., 2019, MNRAS, 490, 319

Nyland K. et al., 2017, ApJ, 845, 50

Onishi K., Iguchi S., Sheth K., Kohno K., 2015, ApJ, 806, 39

Onishi K., Iguchi S., Davis T. A., Bureau M., Cappellari M., Sarzi M., Blitz L., 2017, MNRAS, 468, 4663

Osterbrock D. E., 1989, S\&T, 78, 491

Pacucci F., Natarajan P., Volonteri M., Cappelluti N., Urry C. M., 2017, ApJ, 850, L42

Pacucci F., Loeb A., Mezcua M., Martín-Navarro I., 2018, ApJ, 864, L6

Panessa F., Bassani L., Cappi M., Dadina M., Barcons X., Carrera F. J., Ho L. C., Iwasawa K., 2006, A\&A, 455, 173

Pechetti R., Seth A., Neumayer N., Georgiev I., Kacharov N., den Brok M., 2020, ApJ, 900, 32

Planck Collaboration XVI, 2014, A\&A, 571, A16

Pogge R. W., Eskridge P. B., 1993, AJ, 106, 1405

Prandoni I., Laing R. A., Parma P., de Ruiter H. R., Montenegro-Montes F. M., Wilson T. L., 2007, A Search for Molecular Gas in Low-Luminosity Radio Galaxies. p. 271

Prandoni I., Laing R. A., de Ruiter H. R., Parma P., 2010, A\&A, 523, A38

Press M., 2007, Sci. Am., 297, 100

Raimundo S. I., Davies R. I., Canning R. E. A., Celotti A., Fabian A. C., Gandhi P., 2017, MNRAS, 464, 4227

Reines A. E., Comastri A., 2016, Publ. Astron. Soc. Aust., 33, e054

Reines A. E., Volonteri M., 2015, ApJ, 813, 82

Reines A. E., Sivakoff G. R., Johnson K. E., Brogan C. L., 2011, Nature, 470, 66

Reines A. E., Greene J. E., Geha M., 2013, ApJ, 775, 116

Riffel R. A., Storchi-Bergmann T., Winge C., 2013, MNRAS, 430, 2249

Riffel R. A., Storchi-Bergmann T., Riffel R., 2015, MNRAS, 451, 3587

Roediger J. C., Courteau S., 2015, MNRAS, 452, 3209

Rubin V. C., Burstein D., Ford W. K. J., Thonnard N., 1985, ApJ, 289, 81

Saglia R. P. et al., 2016, ApJ, 818, 47

Sahu N., Graham A. W., Davis B. L., 2019a, ApJ, 876, 155

Sahu N., Graham A. W., Davis B. L., 2019b, ApJ, 887, 10

Salo H. et al., 2015, ApJS, 219, 4

Sandage A., Bedke J., 1994, in Sandage A., Bedke J., eds, The Carnegie Atlas of Galaxies. Vol. 638, Carnegie Inst of Washington

Sandage A., Tammann G. A., 1981, A Revised Shapley-Ames Catalog of Bright Galaxies. Carnegie Inst of Washington

Sani E. et al., 2018, Frontiers Astron. Space Sci., 5, 2

Satyapal S., Böker T., Mcalpine W., Gliozzi M., Abel N. P., Heckman T., 2009, ApJ, 704, 439

Savorgnan G. A. D., Graham A. W., Marconi A., Sani E., 2016, ApJ, 817, 21

Schawinski K., Thomas D., Sarzi M., Maraston C., Kaviraj S., Joo S.-J., Yi S. K., Silk J., 2007, MNRAS, 382, 1415

Schlafly E. F., Finkbeiner D. P., 2011, ApJ, 737, 103

Schödel R., Merritt D., Eckart A., 2009, A\&A, 502, 91 
Scott N., Graham A. W., Schombert J., 2013, ApJ, 768, 76

Seth A., Agüeros M., Lee D., Basu-Zych A., 2008a, ApJ, 678, 116

Seth A. C., Blum R. D., Bastian N., Caldwell N., Debattista V. P., 2008b, ApJ, 687, 997

Seth A. C. et al., 2010, ApJ, 714, 713

Seth A. C. et al., 2014, Nature, 513, 398

Shankar F., Salucci P., Granato G. L., De Zotti G., Danese L., 2004, MNRAS, 354,1020

She R., Ho L. C., Feng H., 2017a, ApJ, 835, 223

She R., Ho L. C., Feng H., 2017b, ApJ, 842, 131

Shlosman I., 2001, Dynamics of the Central kpc in Barred Galaxies: Theory and Modeling. Cambridge Univ. Press, Cambridge, p. 55

Shlosman I., 2005, in Hüttmeister S., Manthey E., Bomans D., Weis K., eds, AIP Conf. Ser. Vol. 783, The Evolution of Starbursts. Am. Inst. Phys., New York, p.223

Smith M. D. et al., 2019, MNRAS, 485, 4359

Smith M. D. et al., 2021a, MNRAS, 500, 1933

Smith M. D. et al., 2021b, MNRAS, 503, 5984

Spitzer L., 1978, Physical Processes in the Interstellar Medium. Wiley \& Sons Inc

Stierwalt S., Haynes M. P., Giovanelli R., Kent B. R., Martin A. M., Saintonge A., Karachentsev I. D., Karachentseva V. E., 2009, AJ, 138, 338

Stone N. C., Metzger B. D., 2016, MNRAS, 455, 859

Stone N. C., Küpper A. H. W., Ostriker J. P., 2017, MNRAS, 467, 4180

Storchi-Bergmann T., Schnorr-Müller A., 2019, Nat. Astron., 3, 48

Thater S., 2019, ALMA2019: Science Results and Cross-Facility Synergies. p. 129

Thater S. et al., 2017, A\&A, 597, A18

Thater S., Krajnović D., Cappellari M., Davis T. A., de Zeeuw P. T., McDermid R. M., Sarzi M., 2019, A\&A, 625, A62

Thater S., Krajnović D., Nguyen D. D., Iguchi S., Weilbacher P. M., 2020, in Valluri M., Sellwood J. A., eds, Galactic Dynamics in the Era of Large Surveys, Vol 353. p. 199

Thornton C. E., Barth A. J., Ho L. C., Rutledge R. E., Greene J. E., 2008, ApJ, 686, 892

Valluri M., Ferrarese L., Merritt D., Joseph C. L., 2005, ApJ, 628, 137

van de Ven G., Fathi K., 2010, ApJ, 723, 767

van den Bosch R. C. E., de Zeeuw P. T., 2010, MNRAS, 401, 1770

van den Bosch R. C. E., van de Ven G., 2009, MNRAS, 398, 1117

van den Bosch R. C. E., Greene J. E., Braatz J. A., Constantin A., Kuo C.-Y., 2016, ApJ, 819, 11

van Wassenhove S., Volonteri M., Walker M. G., Gair J. R., 2010, MNRAS, 408, 1139

Verolme E. K. et al., 2002, MNRAS, 335, 517

Verschuur G. L., Schmelz J. T., 2016, ApJ, 832, 98

Vika M., Driver S. P., Graham A. W., Liske J., 2009, MNRAS, 400, 1451

Voggel K. T. et al., 2018, ApJ, 858, 20

Voggel K. T., Seth A. C., Baumgardt H., Mieske S., Pfeffer J., Rasskazov A., 2019, ApJ, 871, 159

Volonteri M., 2010, A\&A Rev., 18, 279

Volonteri M., 2012, in Umemura M., Omukai K., eds, AIP Conf. Ser. Vol. 1480, The First Massive Black Holes. Am. Inst. Phys., New York. p. 289

Volonteri M., Bellovary J., 2012, Rep. Prog. Phys., 75, 124901

Volonteri M., Lodato G., Natarajan P., 2008, MNRAS, 383, 1079

Wada K., Norman C. A., 2002, ApJ, 566, L21

Wiklind T., Henkel C., 1992, A\&A, 257, 437

Woo J.-H., Le H. A. N., Karouzos M., Park D., Park D., Malkan M. A., Treu T., Bennert V. N., 2018, ApJ, 859, 138
Woo J.-H., Cho H., Gallo E., Hodges-Kluck E., Le H. A. N., Shin J., Son D., Horst J. C., 2019, Nat. Astron., 3, 755

Young L. M., 2002, AJ, 124, 788

Young L. M., Bureau M., Cappellari M., 2008, ApJ, 676, 317

Young L. M. et al., 2011, MNRAS, 414, 940

Young T., Jerjen H., López-Sánchez Á. R., Koribalski B. S., 2014, MNRAS, 444, 3052

Zhang W. M., Soria R., Zhang S. N., Swartz D. A., Liu J. F., 2009, ApJ, 699, 281

\section{SUPPORTING INFORMATION}

Supplementary data are available at MNRAS online.

Appendix A: Supplementary tables.

Appendix B: Supplementary figures.

Please note: Oxford University Press is not responsible for the content or functionality of any supporting materials supplied by the authors. Any queries (other than missing material) should be directed to the corresponding author for the article.

${ }^{1}$ Department of Physics, International University - Quarter 6, Linh Trung Ward, Thu Duc City, Ho Chi Minh City, Vietnam

${ }^{2}$ Vietnam National University - Quarter 6, Linh Trung Ward, Thu Duc City, Ho Chi Minh City, Vietnam

${ }^{3}$ Sub-department of Astrophysics, Department of Physics, University of Oxford, Denys Wilkinson Building, Keble Road, Oxford OX1 3RH, UK

${ }^{4}$ Yonsei Frontier Lab and Department of Astronomy, Yonsei University, 50 Yonsei-ro, Seodaemun-gu, Seoul 03722, Republic of Korea

${ }^{5}$ Department of Astrophysics, University of Vienna, Türkenschanzstrasse 17, 1180 Wien, Austria

${ }^{6}$ National Research Council, Resident at the Naval Research Laboratory, Washington, DC 20375, USA

${ }^{7}$ Leibniz-Institut für Astrophysik Potsdam (AIP), An der Sternwarte 16, D14482 Potsdam, Germany

${ }^{8}$ School of Physics and Astronomy, Cardiff University, Queens Buildings, The Parade, Cardiff CF24 3AA, UK

${ }^{9}$ Department of Astrophysics, Princeton University, Princeton, NJ 08540, USA

${ }^{10}$ Max Planck Institut für Astronomie (MPIA), Königstuhl 17, D-69121 Heidelberg, Germany

${ }^{11}$ National Astronomical Observatory of Japan (NAOJ), National Institute of Natural Sciences (NINS), 2-21-1 Osawa, Mitaka, Tokyo 181-8588, Japan

${ }^{12}$ Department of Astronomical Science, The Graduate University for Advanced Studies (SOKENDAI), 2-21-1 Osawa, Mitaka, Tokyo 181-8588, Japan

${ }^{13}$ Nućleo de Astronomía de la Facultad de Ingeniería, Universidad Diego Portales, Av. Ejéercito Libertador 441, Santiago, Chile

${ }^{14}$ Department of Physics, Quy Nhon University, 170 An Duong Vuong Quy Nhon, 55111, Vietnam

${ }^{15}$ LESIA, Observatoire de Paris, Université PSL, CNRS, Sorbonne Université, Univ. Paris Diderot, Sorbonne Paris Cité, 5 place Jules Janssen, F-92195 Meudon, France

${ }^{16}$ Training Management Office, Mientrung University of Civil Engineering, Ha Huy Tap, Tuy Hoa, Phu Yen, Vietnam

This paper has been typeset from a $\mathrm{T}_{\mathrm{E}} \mathrm{X} / \mathrm{ET} \mathrm{E} \mathrm{X}$ file prepared by the author. 